\title{
The calcium binding protein S100 $\beta$ marks hedgehog-responsive resident vascular stem cells within vascular lesions
}

\author{
Mariana Di Luca ${ }^{1,4}$, Emma Fitzpatrick ${ }^{1,4}$, Denise Burtenshaw ${ }^{1,4}$, Weimin Liu ${ }^{2,4}$, Jay-Christian Helt ${ }^{2}$, Roya Hakimjavadi ${ }^{1}$, Eoin Corcoran ${ }^{1}$ \\ Yusof Gusti ${ }^{1}$, Daniel Sheridan (iD), Susan Harman ${ }^{1}$, Catriona Lally ${ }^{3}$, Eileen M. Redmond (iD ${ }^{2,5}$ and Paul A. Cahill (iD) ${ }^{1,5 \times}$ \\ A hallmark of subclinical atherosclerosis is the accumulation of vascular smooth muscle cell (SMC)-like cells leading to intimal \\ thickening. While medial SMCs contribute, the participation of hedgehog-responsive resident vascular stem cells (vSCs) to lesion \\ formation remains unclear. Using transgenic eGFP mice and genetic lineage tracing of S100 $\beta$ vSCs in vivo, we identified S100 $\beta$ / \\ Sca1 cells derived from a S100 $\beta$ non-SMC parent population within lesions that co-localise with smooth muscle a-actin (SMA) cells \\ following iatrogenic flow restriction, an effect attenuated following hedgehog inhibition with the smoothened inhibitor, \\ cyclopamine. In vitro, $\mathrm{S} 100 \beta / \mathrm{Sca} 1$ cells isolated from atheroprone regions of the mouse aorta expressed hedgehog signalling \\ components, acquired the di-methylation of histone 3 lysine $4(\mathrm{H} 3 \mathrm{~K} 4 \mathrm{me} 2)$ stable SMC epigenetic mark at the Myh11 locus and \\ underwent myogenic differentiation in response to recombinant sonic hedgehog (SHh). Both S100 $\beta$ and PTCH1 cells were present \\ in human vessels while $S 100 \beta$ cells were enriched in arteriosclerotic lesions. Recombinant SHh promoted myogenic differentiation \\ of human induced pluripotent stem cell-derived $S 100 \beta$ neuroectoderm progenitors in vitro. We conclude that hedgehog- \\ responsive $S 100 \beta$ vSCs contribute to lesion formation and support targeting hedgehog signalling to treat subclinical \\ arteriosclerosis.
}

npj Regenerative Medicine (2021)6:10; https://doi.org/10.1038/s41536-021-00120-8

\section{INTRODUCTION}

Atherosclerosis is a chronic progressive inflammatory disease that leads to myocardial infarction and stroke ${ }^{1}$. Pathologic observations in humans vessels confirm that early 'transitional' lesions enriched with smooth muscle (SMC)-like cells called adaptive lesions are routinely present in atherosclerotic-prone regions of arteries before pathologic intimal thickening, lipid retention, and the appearance of a developed plaque ${ }^{2}$. Endothelial cell (EC) dysfunction due to disturbed blood flow is classically associated with the development of atheroprone lesions ${ }^{3}$, while the embryological origin (e.g. neuroectoderm versus mesoderm) of SMCs may also influence disease localisation and progression ${ }^{4}$. These early lesions are routinely modelled in mouse carotid arteries following flow restriction ${ }^{5}$ and can further rapidly develop into advanced atherosclerotic plaques in ApoE knockout on a Western diet ${ }^{6,7}$.

Lineage tracing and single-cell RNA sequence analysis (scRNAseq) have provided compelling evidence in various animal models for the involvement of a rare population of (i) differentiated myosin heavy chain 11 (Myh11) medial SMC that are stem cell antigen-1 positive $\left(\mathrm{Sca}^{+}\right)^{8,9}$ and (ii) various adventitial and medial progenitors in progressing intimal thickening ${ }^{10-13}$. While some adventitial progenitors are derived from a parent Myh11 SMC population $^{14}$, non-SMC derived SRY-related HMG-box 10 (Sox10) adventitial/medial resident multipotent vascular stem cells (vSC), glioma-associated oncogene homolog 1 (Gli), and Nestin progenitor cells have also been implicated ${ }^{13,15}$. Despite these insights, the putative role of resident vSCs that do not originate from medial Myh11 ${ }^{+}$SMCs in promoting intimal thickening remains controversial.
$\mathrm{S} 100 \beta$, a member of the $\mathrm{S} 100$ multigenic family expressing small ( $9 \mathrm{kDa}$ and $14 \mathrm{kDa}$ ) Ca2+-binding proteins of the EF-hand type, is primarily localised to astrocytes and glial cells under normal physiological conditions and is positively associated with age and various neurodegenerative and neuroinflammatory diseases $^{16}$. S100 $\beta$ promotes intimal thickening through interaction with the receptor for advanced glycation end-products (RAGE) ${ }^{17}$ and induces migration and infiltration of inflammatory cells ${ }^{18,19}$. $S 100 \beta$ plays a crucial role in maintaining an intermediate state of Sca1 progenitor cells following injury-induced neointimal thickening through a RAGE and stromal cell-derived factor-1 a/ CXCR4 signalling mechanism ${ }^{20}$.

Hedgehog signalling $(\mathrm{Hh})$ is essential for normal embryonic development and plays a pivotal role in the maintenance of adult progenitor/stem cells in tissue repair after injury ${ }^{21}$. Hh signalling is initiated upon binding of a ligand to the canonical receptors, PTCH1 and PTCH2 (Patched 1 and 2) ${ }^{22}$, which leads to disinhibition of SMO (Smoothened) and activation of a complex signalling cascade that regulates the GLI (glioma-associated oncogene homolog 1) family zinc finger transcription factors $\left(G L I 1, G L I 2\right.$, and GLI3) ${ }^{23}$. GLI activators induce the transcription of $\mathrm{Hh}$ target genes primarily involved in cell proliferation, cell survival, and cell fate specification, including PTCH and GLI ${ }^{24,25}$. HHIP (hedgehog interacting protein) modulates hedgehog signalling activity by binding and inhibiting the action of hedgehog proteins ${ }^{26}$. Genome wide association studies (GWAS) in coronary artery disease patients have identified variants at the 14q32 HHIPL1 (Hedgehog interacting protein-like 1) that encode a paralog of $\mathrm{HHIP}^{27}$ to increase $\mathrm{Hh}$ signalling and regulate SMC proliferation and migration in vitro ${ }^{28}$. In atherosclerotic mice, $\mathrm{Hh}$ has a permissive role through increased lipid uptake by

\footnotetext{
${ }^{1}$ Dublin City University, Vascular Biology \& Therapeutics Group, School of Biotechnology, Dublin, Ireland. ${ }^{2}$ University of Rochester, Department of Surgery, Rochester, NY, USA. ${ }^{3}$ Trinity College Dublin, Trinity Centre for Bioengineering, Trinity Biomedical Sciences Institute, Dublin, Ireland. ${ }^{4}$ These author contributed equally: Mariana Di Luca, Emma Fitzpatrick, Denise Burtenshaw, Weimin Liu. ${ }^{5}$ These authors jointly supervised this work: Eileen M. Redmond, Paul A. Cahill. ${ }^{凶}$ email: paul.cahill@dcu.ie
} 
macrophages ${ }^{29}$, while global Hhipl1 deletion reduces plaque burden $^{28}$. Hh signalling, especially SHh and signal peptide CUB domain and EGF like domain containing 2 (Scube2), are overexpressed in injured arteries during lesion formation after flow restriction ${ }^{30-32}$, autogenous vein grafts ${ }^{33}$, and hypoxic pulmonary arterial $\mathrm{SMCs}^{34}$. Adventitial $\mathrm{Sca}^{+}{ }^{+}$cells that co-localise with $\mathrm{SHh}$ and Ptch $1^{35}$ significantly contribute to intimal thickening in vivo $31,36,37$ while $\mathrm{Hh}$ inhibition with cyclopamine or local perivascular depletion of Ptch1 attenuates intimal thickening following iatrogenic flow restriction in vivo ${ }^{38,39}$.

Given the pivotal role of $\mathrm{Hh}$ in the maintenance of adult progenitor/stem cells, tissue repair, and atherosclerosis ${ }^{21,28}$, we sought to determine the role of Hh-responsive S100 $3 / S c a 1$ vSCs and their myogenic progeny in contributing to intimal thickening and the progression of adaptive lesions in vivo and, furthermore, whether $\mathrm{Hh}$ signalling directs murine $\mathrm{S} 100 \beta$ vSCs and hiPSCderived $S 100 \beta$ neuroectoderm stem cells to undergo proliferation and myogenic differentiation in vitro.

\section{RESULTS}

\section{Perivascular Sca1 and S100 $\beta$ stem vSCs are present in normal} adult vessels

The presence of perivascular Sca1 and $\mathrm{S} 100 \beta$ vSCs was first confirmed in whole mounted vessels from Sca1-eGFP and S100ß-Cre-tdT mice. A discrete number of Sca1-eGFP and S100 $\beta$-tdT cells were present on collagen fibres within the adventitial layer of the thoracic aorta, when compared to wildtype (WT) controls [Supplementary Fig. 1a-d]. The cells were located predominantly at the aortic arch and at bifurcations with intercostal vessels, and their presence diminished along the descending and abdominal aorta [Supplementary Fig. 1b, c]. When the media was removed, Sca1-eGFP cells were clearly present in adventitial layer of the thoracic aorta [Supplementary Fig. 1d] and in perivascular regions of the carotid artery [Supplementary Fig. 1d].

\section{Sca1 and $S 100 \beta$ cells are present in intimal lesions from murine carotid arteries following iatrogenic flow restriction}

The contribution of Sca1 cells to intimal medial thickening following iatrogenic flow restriction was first assessed in Sca1eGFP transgenic mice following partial ligation of the left carotid artery (LCA). Vessels were harvested on day 3, 7, and 14 days postligation and paraformaldehyde fixed and paraffin-embedded cross sections were evaluated for eGFP (indicative of Sca1 expression) by confocal microscopy. Morphometric analysis confirmed that adventitial and intimal volumes increased significantly over time following ligation, without any significant change to medial volume, concomitant with a significant reduction in luminal volume after 7 and 14 days [Fig. 1a].

The number of cells within the adventitial, medial and intimal layers of the LCA did not significantly change $3 \mathrm{~d}$ post-ligation [Fig. 1b-d]. No Sca1- eGFP cells were visible within the medial layer of sham vessels which was populated primarily by a-smooth muscle actin (SMA) SMCs [Fig. 1e]. While a small but significant number of Sca1-eGFP cells were present within the adventitial and intimal layers of sham vessels, the fraction of Sca1-eGFP cells significantly increased within the intimal layer $3 \mathrm{~d}$ post-ligation [Fig. 1e, f]. Immunohistochemical analysis using anti-eGFP confirmed the presence of intimal Sca1-eGFP cells in ligated vessels [Fig. 1e] and further demonstrated that intimal Sca1-GFP cells co-express PECAM$1 /$ CD31 and endothelial cell nitric oxide (eNOS) [Fig. 1e]. Moreover, there was also a notable increase in the expression of the proapoptotic protein, Bax within the intimal, adventitial and medial layer of the LCA and the medial layer of the RCA $3 \mathrm{~d}$ post-ligation [Fig. 1g, $\mathrm{h}]$, consistent with disturbed flow- and strain -induced apoptosis ${ }^{40}$.

While the majority of Sca1-eGFP cells were present in the adventitial and intimal layers of sham-operated vessels, a small fraction were also visible within the medial layer after 7,14 , and 21d [Fig. 2a, b, g and Supplementary Fig. 2a, b]. Upon injury, there was a notable appearance of Sca1-eGFP cells within the expanding intimal layer of ligated vessels after 7, 14, and 21d [Fig. 2a, b, d, g] that coincided with an increase in the number of cells [Fig. $2 c$ ] and the volume of the neointimal layer [Fig. 1a]. Cumulative analysis revealed that there was a significant increase in the fraction of Sca1-eGFP cells within neointimal layers after 7 and 14 and $21 \mathrm{~d}$, respectively, when compared to sham vessels or contralateral RCA [Fig. 2d, h]. The majority of Sca1 cells within these lesions co-localised with $\mathrm{SMA}^{+}$cells [Fig. 2e, f, Supplementary Fig. 3a].

The contribution of $\mathrm{S} 100 \beta$ vSC to intimal thickening following iatrogenic flow restriction was also assessed in $5100 \beta$-eGFP transgenic mice. While the majority of $\mathrm{S} 100 \beta$-eGFP cells were present in the adventitial layer of sham-operated vessels and the contralateral RCA, a small fraction were also present within the medial layer after 21d [Supplementary Fig. 2a, c, Fig. 2g, h]. In contrast, no intimal S100 $\beta$-eGFP cells were present in sham vessels or the contralateral RCA [Supplementary Fig. 2a, c-e]. Upon injury, the fraction of $\mathrm{S} 100 \beta-\mathrm{eGFP}$ cells within lesions significantly increased when compared to sham vessels and the contralateral RCA [Supplementary Fig. 2d, e and Fig. 2g, h]. Importantly, the number of double positive Sca1/S100 $\beta$ cells increased within the neointimal layers of ligated vessels compared to the sham [Supplementary Fig. 2d, e] and contralateral RCA control [Fig. 2g, h]. Similarly, the number of double positive S100B/SMA cells increased within lesions compared to the contralateral RCA [Fig. 2i, j]. Taken together, these data suggest double positive Sca1/S100 $\beta$ cells populate vascular lesions following iatrogenic injury in vivo.

\section{Lineage tracing analysis of marked perivascular $\mathrm{S} 100 \beta$ cells iatrogenic flow restriction}

In order to determine the source of intimal double positive Sca1/ S100 $\beta$ cells following flow restriction, lineage tracing analysis was performed using transgenic S100 $\beta$-eGFP-CreERT2-Rosa26-tdTomato reporter mice [Supplementary Fig. 4a]. As cre-mediated recombination results in a permanent heritable change in a cell's genome, these cells and their progeny remain tdTomato (tdT) positive. The animals were treated with tamoxifen (Tm) for 7 days to induce nuclear translocation of CreER(Tm) and subsequent recombination to indelibly mark $S 100 \beta$ cells with red fluorescent tdT seven days before ligation was performed [Supplementary Fig. 4b]. Only S100 $\beta$ cells present during the period of $\mathrm{Tm}$ treatment and their subsequent progeny following injury are marked with tdT. The tissue specificity and recombination efficiency of the Tm-induced Cre activity was confirmed in bone marrow smears and neuronal tissue from S100ß-eGFP-CreERT2-Rosa26-tdTomato mice. Treatment with $\mathrm{Tm}$ resulted in Cre-mediated recombination and expression of S100 $\beta$ - tdT in $>90 \%$ of neuronal cells of the carotid sinus nerve (Hering's nerve) without any S100ß-tdT cells observed in the bone marrow smears [Supplementary Fig. 4c]. The neuronal staining was confirmed by immunohistochemical analysis of $S 100 \beta$ expression using both anti-S100 $\beta$ and anti-tdT antibodies (data not shown).

Morphometric analysis of the S100ß-eGFP-creER2-Rosa26tdT mice confirmed significant intimal thickening in transgenic mice following ligation injury when compared to the sham LCA and contralateral RCA [Fig. 3a-c]. Treatment of S100ß-eGFP-creER2Rosa26tdT transgenic mice with tamoxifen indelibly marked S100 $\beta$ cells $(S 100 \beta$-tdT) within the adventitial layer of the sham LCA prior to flow restriction [Fig. 3d]. No cells are marked when these double transgenic mice were treated with the vehicle control (corn oil) [Fig. 3d]. Importantly, no S100 $\beta$-tdT cells were observed in the intimal (EC) or medial (SMC) layers of these sham vessels following tamoxifen treatment [Fig. 3d]. However, following ligation, there was a striking increase in the number of S100ß-tdT marked cells within the medial and intimal layers of the 

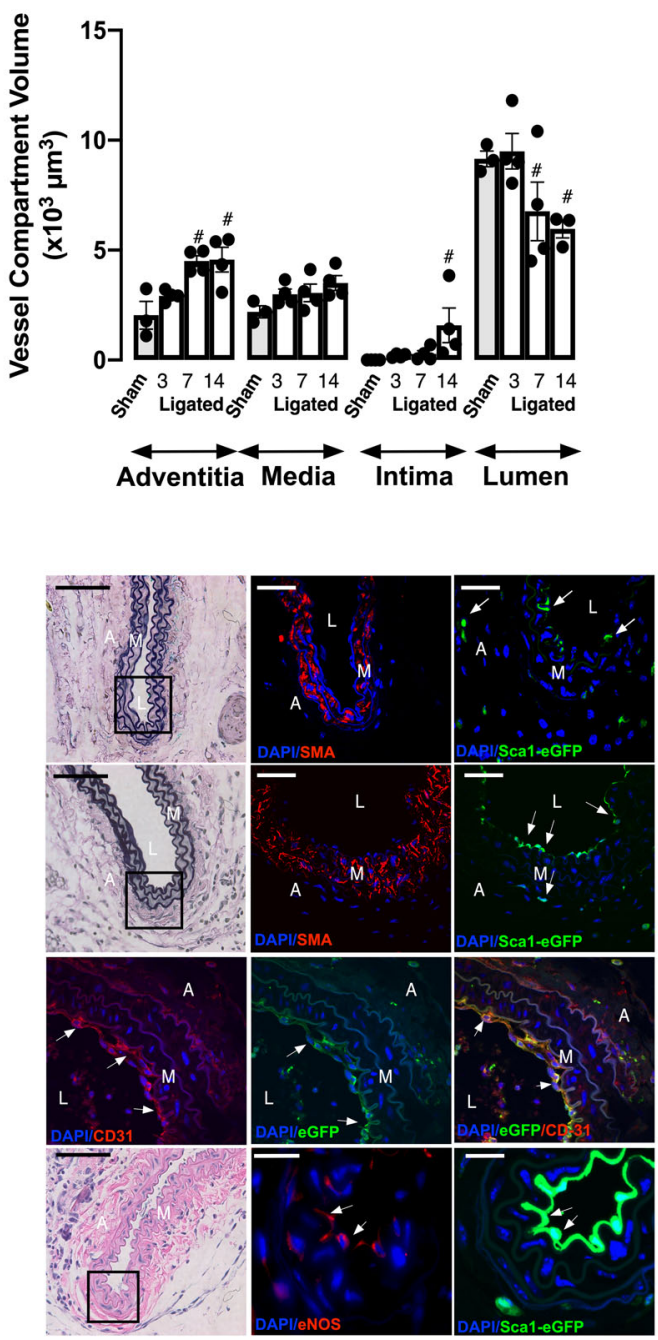

f

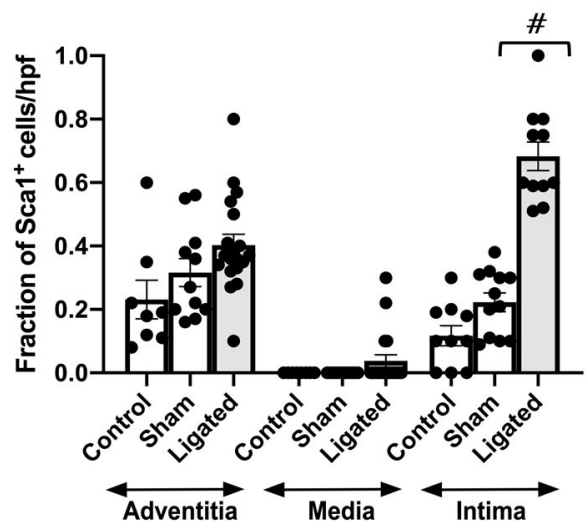

b

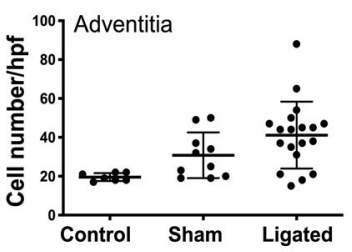

C

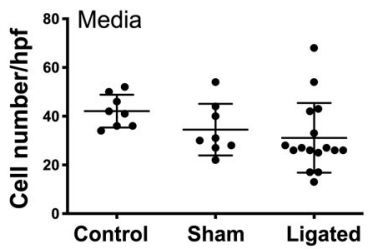

d

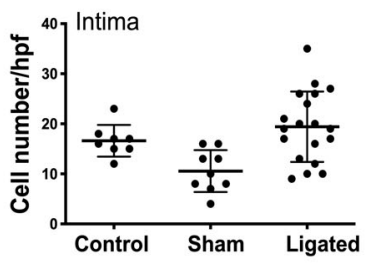

9

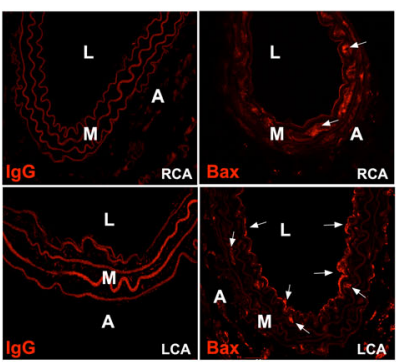

Ligated (3d)

h

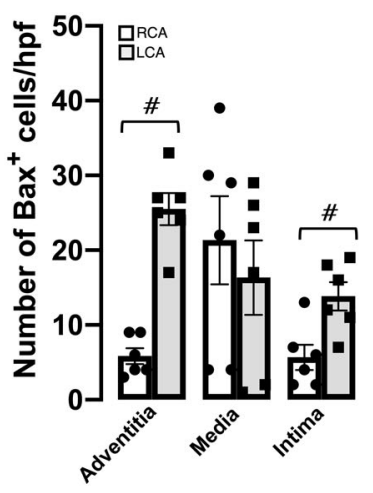

Fig. 1 Expression of Sca1-eGFP and S100ß-eGFP cells following iatrogenic flow restriction after 3 days. a Morphometric analysis of adventitial, medial, intimal and luminal volumes following partial ligation of the left carotid artery compared to sham controls. Data are the mean \pm SEM of four animals, ${ }^{\#} p<0.05$ vs sham. $\mathbf{b}$-d The number of DAPI nuclei/hpf in cross sections of $\mathbf{b}$ adventitia, $\mathbf{c}$ media, and $\mathbf{d}$ intima following partial ligation of the left carotid artery (LCA) after $3 \mathrm{~d}$ compared to untouched (control) and sham. Data are the mean \pm SEM of 3-5 representative images per animals, $n=3,{ }^{\#} p<0.05$. e Verhoeff-Van Gieson stained sections and corresponding confocal fluorescence images of DAPI nuclei (blue), Sca1-eGFP (green), and immunofluorescence of $\alpha$-actin (SMA) (red), anti-eGFP (green), anti-CD31 (red) and anti-eNOS (red) expression in ligated LCA vessels expression in sham-operated and ligated LCA vessels. Arrows highlight Sca1-eGFP expressing cells in the adventitia (A), media (M) and neointima (NI) and CD31 and eNOS in intima. Scale bar $=20 \mu \mathrm{m}$. $\mathbf{f}$ The fraction of Sca1 cells/high power field (hpf) in the adventitia, media, and intima of sham and ligated vessels after 3 days. Data are the mean \pm SEM of $3-5$ sections, \# $p \leq 0.05$ from a minimum of four animals per experimental group. $\mathbf{g}$ Representative immunofluorescence images for the pro-apoptotic marker, Bax, (red) in ligated left carotid artery (LCA) vessels and contralateral right carotid artery (RCA) after $3 \mathrm{~d}$. IgG was used as a control. $\mathbf{h}$ The number of Bax cells/ hpf in the adventitia, media, and intima of sham and ligated vessels after $3 \mathrm{~d}$. Arrows highlight Bax expressing cells within the adventitia (A), media (M), and neointima (NI). Data are the mean \pm SEM of 3-6 sections per experimental group from three animals. ${ }^{\#} p \leq 0.05$. Scale bar $=20 \mu$ m. 
a

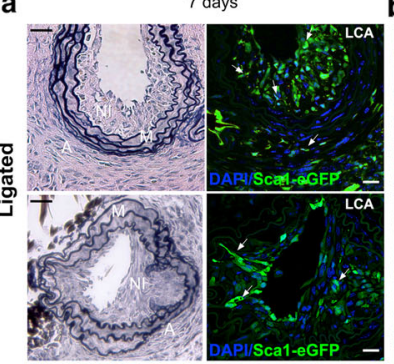

b

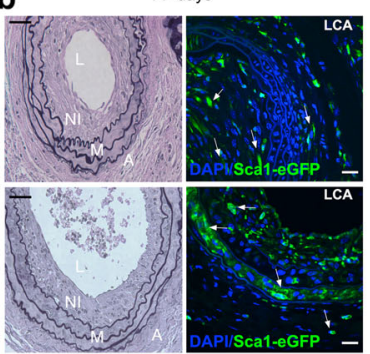

C
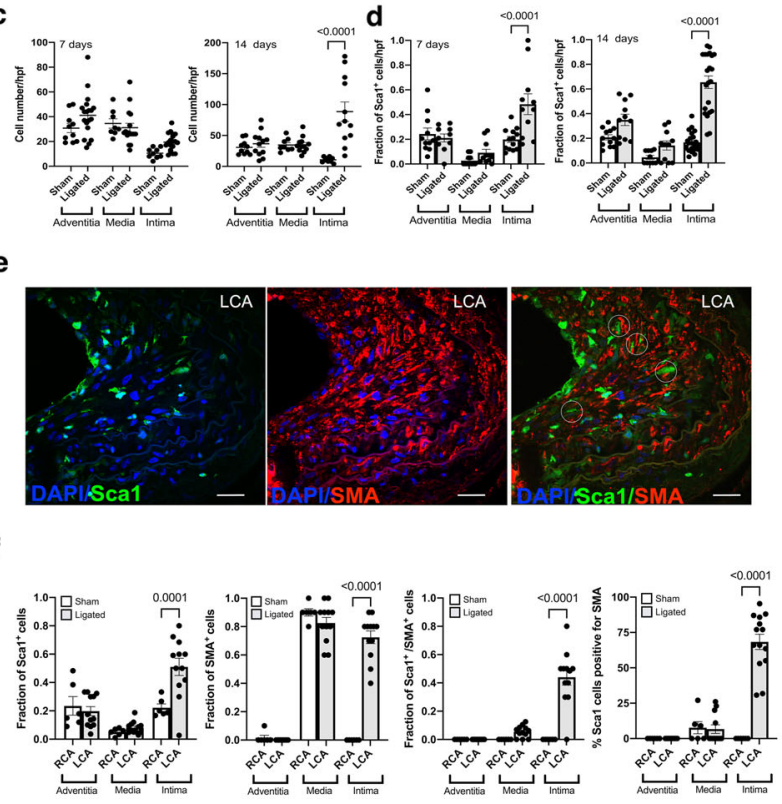

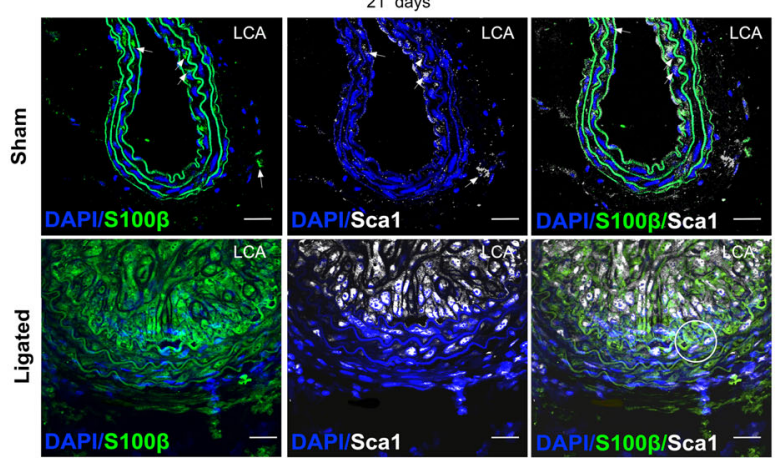

h
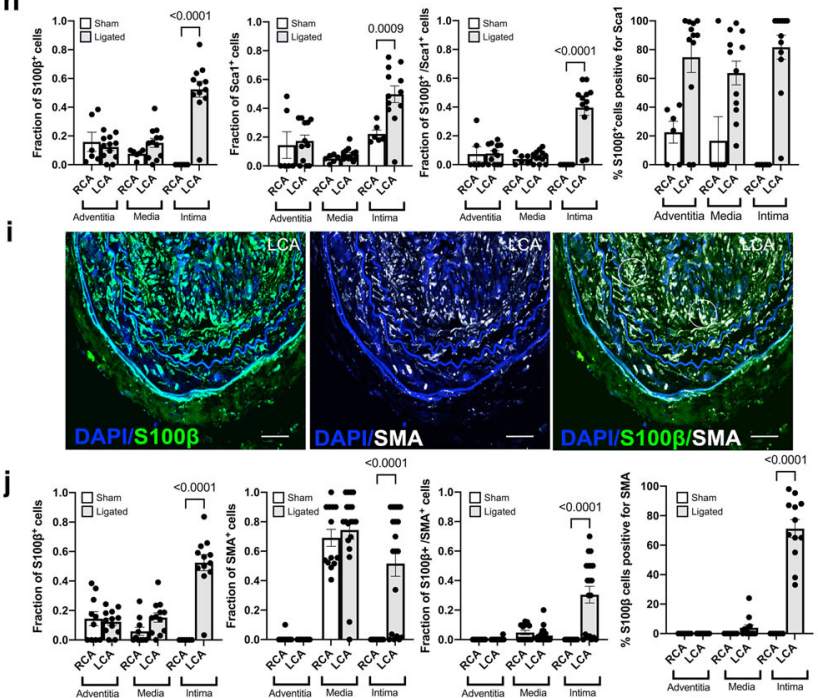

Fig. 2 Expression of Sca1-eGFP and S100ß-eGFP cells following iatrogenic flow restriction after 7, 14, and 21 days. a, b Representative Verhoeff-Van Gieson stained sections and corresponding confocal fluorescence images of DAPI nuclei (blue) and Sca1-eGFP (green) expression in LCA after $\mathbf{a} 7$ days and $\mathbf{b} 14$ days. Scale bar $=20 \mu \mathrm{m}$. c The number of DAPI nuclei/hpf in cross sections of adventitia, media and intima following partial ligation of the LCA after 7 and 14 days compared to sham controls. Data are the mean \pm SEM of 3-5 representative images per animal, $n=4$. d The fraction of Sca1 cells/high power field (hpf) in the adventitia, media, and intima of sham and ligated vessels after 7 days and 14 days. Data are the mean \pm SEM of 10-15 sections from four animals per experimental group. e Representative confocal fluorescence images of DAPI nuclei (blue), immunofluorescence of anti-eGFP (green) expression and anti-SMA (Red) in the LCA 14 days postligation in Sca1-eGFP mice. Data are representative of five images per animal. f Cumulative analysis of Sca $1^{+}$cells, SMA ${ }^{+}$cells, double stained Sca1/SMA-positive cells and the percentage of Sca $1^{+}$cells that were SMA $1^{+}$in the adventitial, medial and intimal layer of RCA and LCA vessels after 21 days, respectively. Data are the mean \pm SEM, $n=15-20$ sections from 5 animals. g Representative confocal fluorescence images of DAPI nuclei (blue), immunofluorescence of anti-eGFP (green) expression and anti-Sca1 (far red white) in sham and ligated LCA after 21 days in

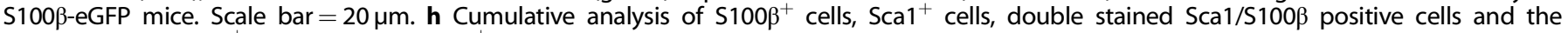
percentage of $S 100 \beta^{+}$cells that were Sca $1^{+}$in the adventitial, medial and intimal layer of RCA and LCA vessels, respectively after 21 days in S100 $\beta$-eGFP mice. Data are the mean \pm SEM, $n=15-20$ sections from 5 animals/group. i. Representative confocal fluorescence images of DAPI nuclei (blue), immunofluorescence of anti-eGFP (green) expression and anti-SMA (far red, white) in the RCA and LCA 21 days post-ligation in S100 $\beta$-eGFP mice. Data are from a minimum of four animals per experimental group. Scale bar $=20 \mu \mathrm{m}$. $\mathbf{j}$ Cumulative analysis of S100 $\beta^{+}$cells, $\mathrm{SMA}^{+}$cells, double stained S100/SMA-positive cells and the percentage of S100 $\beta^{+}$cells that were SMA ${ }^{+}$in the adventitial, medial and intimal layer of RCA and LCA vessels, respectively, after 21 days in S100 $\beta$-eGFP mice. Data are the mean \pm SEM, $n=15-20$ sections from 4 animals/group.

ligated LCA, compared to sham controls [Fig. 3e, f]. In order to rule out the possibility that $\mathrm{Tm}$ treatment continues to label a significant number of cells for weeks after last Tm treatment ${ }^{41}$, we confirmed that the fraction of S100 $\beta$-tdT marked cells remained significantly higher in the neointima of LCA four weeks after the last $\mathrm{Tm}$ injection when compared to the RCA [Supplementary Fig. 4d, e]. Moreover, the fraction of S100 $\beta$-tdT marked cells within the LCA adventitial, medial and intimal layers of ligated vessels after one and four weeks from the last Tm injection was of a similar magnitude [Supplementary Fig. 4f].

When S100 $\beta$-tdT marked cells were evaluated for co-expression of Sca1 and SMA by immunohistochemistry using anti-Sca1, antiSMA and anti-tdT antibodies, S100 $\beta$-tdT marked cells co-localised with Sca1 cells in the adventitial and medial layers (but not the intimal layer) of the control contralateral RCA before injury [Supplementary Fig. 3d, Fig. 3g]. There was a notable increase in the number of double stained Sca1/S100 $\beta$-tdT cells in the media and neointima of the LCA compared to the contralateral RCA [Fig. $3 \mathrm{~g}$ ] and the sham LCA control [Supplementary Fig. 3b] in double stained SMA/S100ß-tdT cells within the neointima and media compared to the sham control [Supplementary Fig. 3c]. Cumulative analysis confirmed both a significant increase in the fraction of $\mathrm{S} 100 \beta$-tdT cells [Fig. 3h] and the fraction of double stained $\mathrm{S} 100 \beta / \mathrm{Sca} 1$ cells [Fig. 3i] within the LCA medial and intimal layers compared to the contralateral RCA control. Moreover, the majority of Sca1 cells within ligated vessels were S100ß-tdT cells [Fig. 3j]. Since intimal and medial cells are not marked with tdT prior to injury, these data suggest that these cells are derived from a non- 

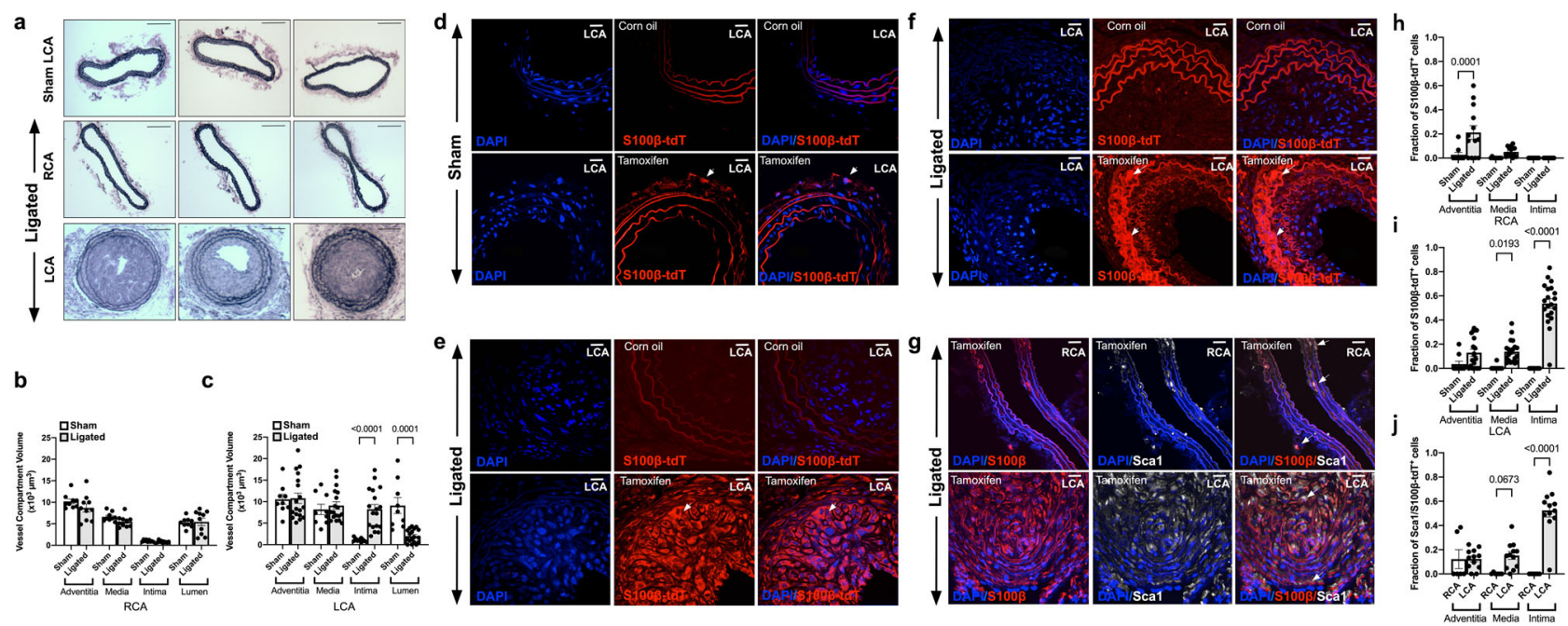

Adventitia Meciai hnima

Fig. 3 Lineage tracing analysis of S100ß-Cre(ERT2)-tdT marked cells following iatrogenic flow restriction for 21 days. a-c Verhoeff-Van Gieson stained sections and morphometric analysis of adventitial, medial, intimal and luminal volumes of the $\mathbf{b}$ contralateral RCA control
compared to the c LCA following ligation for $21 \mathrm{~d}$. Data are the mean \pm SEM of 10 images, $\#<<0.05$ vs sham controls. d-f Representative confocal fluorescence images of DAPI nuclei (blue) and S100 $\beta$-tdT (red) cells in mice treated with corn oil (control) or tamoxifen for 7 days prior to washout for 1 week in $\mathbf{d}$ sham and $\mathbf{e}, \mathbf{f}$ ligated LCA. Data are minimum of four animals per experimental group. Scale bar $=20 \mu \mathrm{m}$. Arrows highlight S100 -tdT (red) marked cells. g Representative confocal fluorescence images of DAPI nuclei (blue), immunofluorescence of anti-tdT (red) and anti-Sca1 (far red, white) expression in the contralateral RCA and LCA 21 days post-ligation. Scale bar $=20 \mu m$. $\mathbf{h}-\mathbf{j}$ Cumulative analysis of the fraction of S100 $\beta$-tdT ${ }^{+}$cells in the $\mathbf{h}$ RCA and $\mathbf{i}$ LCA and $\mathbf{j}$ Sca1/S100 $\beta$ double stained cells in the adventitial, medial and intimal layer of RCA and LCA vessels, respectively, after 21 days in S100ß-CreERT2-tdT mice.

SMC S100 $\beta$ parent population following flow restriction. Moreover, the majority of intimal Sca1 cells are derived from an S100 $\beta$ parent population.

\section{Hedgehog signalling components control intimal thickening following iatrogenic flow restriction}

The effect of systemic administration of the Hedgehog inhibitor cyclopamine $(10 \mathrm{mg} / \mathrm{kg}, \mathbb{P}$, every other day) on neointimal thickening and the accumulation of Sca1-eGFP cells was evaluated. 2-hydroxypropyl- $\beta$-cyclodextrin (H $\beta C D)$ was used as a vehicle control. Vessels were harvested 7 and $14 \mathrm{~d}$ post-ligation and morphometric analysis was performed. Whole-vessel Gli1 and Gli2 mRNA levels were significantly elevated in ligated vessels treated with $\mathrm{H} \beta C D$ when compared to sham controls (Gli1: $6.1 \pm$ 1.3 after $3 \mathrm{~d}$; Gli2:8.1 \pm 0.9 fold after $14 \mathrm{~d}, p \leq 0.05 n=4$ ), an effect that was significantly reduced following cyclopamine treatment (Gli2: $3.2 \pm 0.3$ fold, $n=4, p \leq 0.05$ ). Immunohistochemical analysis revealed that the $\mathrm{Hh}$ receptor, Ptch1, was up-regulated in LCA following injury concomitant with enhanced Gli2 expression in LCA when compared to sham vessels [Fig. 4a]. Gli2 co-localised with Sca1-eGFP expressing cells and was markedly increased in ligated vessels treated with vehicle $(H \beta C D)$ when compared to sham-operated vessels, especially in the neointima and media of the LCA, an effect that was attenuated following $\mathrm{Hh}$ inhibition [Fig. 4a]. Cyclopamine treatment significantly reduced the fraction of Sca1-eGFP expressing cells [Fig. 4b, c] concomitant with reduced intimal thickening $7 \mathrm{~d}$ and $14 \mathrm{~d}$ post-ligation [Fig. 4d, g]. Collectively, these data suggest that intimal thickening is attenuated by systemic administration of the smoothened inhibitor, cyclopamine, concomitant with a reduction in the accumulation of Gli2/Sca1 Hh-responsive cells within the intimal and medial layers.

\section{Isolation and characterisation of resident $S 100 \beta / S c a 1$ vascular} stem cells from murine aorta

The expression of SMC differentiation markers genes, Myh11 and Cnn 1 was enriched in medial segments from both atheroprone aortic arch (AA) and atheroresistant thoracic/abdominal aorta (TA) regions of the mouse aorta, when compared to NE-4C cells and immortalised MOVAS mSMCs in culture [Fig. 5a]. The level of S100 $\beta$ and Sox 10 expression, both markers of resident vascular stem cells ${ }^{12,42}$, was then assessed in AA and TA regions, with or without the adventitia [Fig. 5b, c]. There was significant enrichment of both $S 100 \beta$ and Sox 10 in the AA when compared to the TA. Moreover, the levels were significantly enriched within the adventitia since its removal resulted in a dramatic reduction in the expression of both transcripts. Nevertheless, medial expression of both genes was still observed in the AA region [Fig. 5b, c].

Resident vascular stem cells (vSCs) were subsequently isolated from mouse aortic arch (AA) and thoracic aorta (TA) by enzymatic digestion and sequential plating, and were characterised by flow cytometry, immunocytochemistry, and gene expression for both stem cell markers and SMC differentiation markers. Flow cytometric analysis confirmed that the cells were Sca1 and $\mathrm{S} 100 \beta$ positive when compared to $\mathrm{Sca} 1$ and $\mathrm{S} 100 \beta$ positive control cell lines (i.e. C3H 10T1/2 and NE-4C, respectively) [Fig. 5d]. The vSCs did not enrich for Myh11 or Cnn1 transcripts when compared to fresh aortic tissue [Fig. 5e, f] or express these proteins [Fig. $5 \mathrm{~g}, \mathrm{~h}$ ] but exhibited greater teleomere length as a measure of stemness when compared to freshly isolated aortic SMC and to the immortalised MOVAS SMCs in culture [Fig. 5i]. Immunocytochemical analysis revealed that vSCs from both AA and TA regions were Sca1 positive and expressed neuroectodermal markers $S 100 \beta$, Sox 10 , Sox 17 , and Nestin but were negative for SMC differentiation markers Cnn1 and Myh11 [Fig. $5 \mathrm{~g}$, h]. The vSCs were enriched for neuroectodermal marker genes S100 $\beta$, Sox10, and Nestin [Supplementary Fig. 5a-c], but not for mesoderm markers Kdr, Pax1 and Tbx6 [Supplementary Fig. $5 \mathrm{~d}-\mathrm{f}]$, when compared to SMCs and NE-4 cells in culture. Collectively, these data indicate that neuroectodermal S100 $\beta$ / Sca1 cells are primarily located within the adventitial perivascular region of atheroprone regions of the mouse aorta and can be isolated and expanded in vitro to retain these phenotypic markers. 

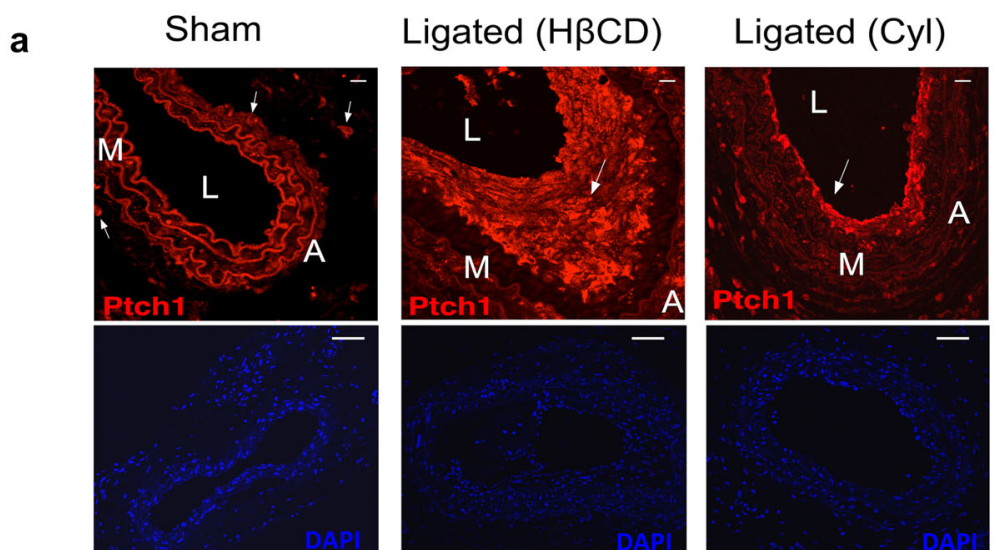

b
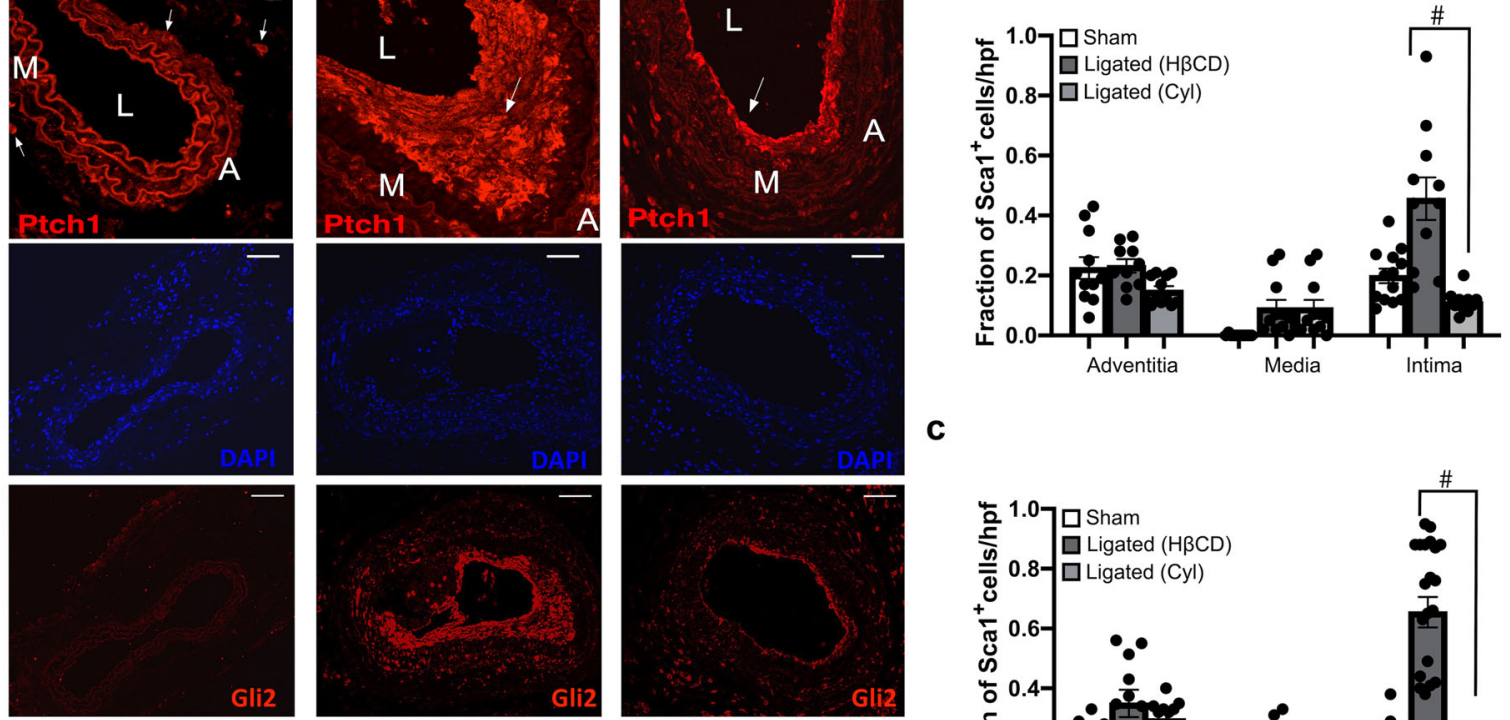

C
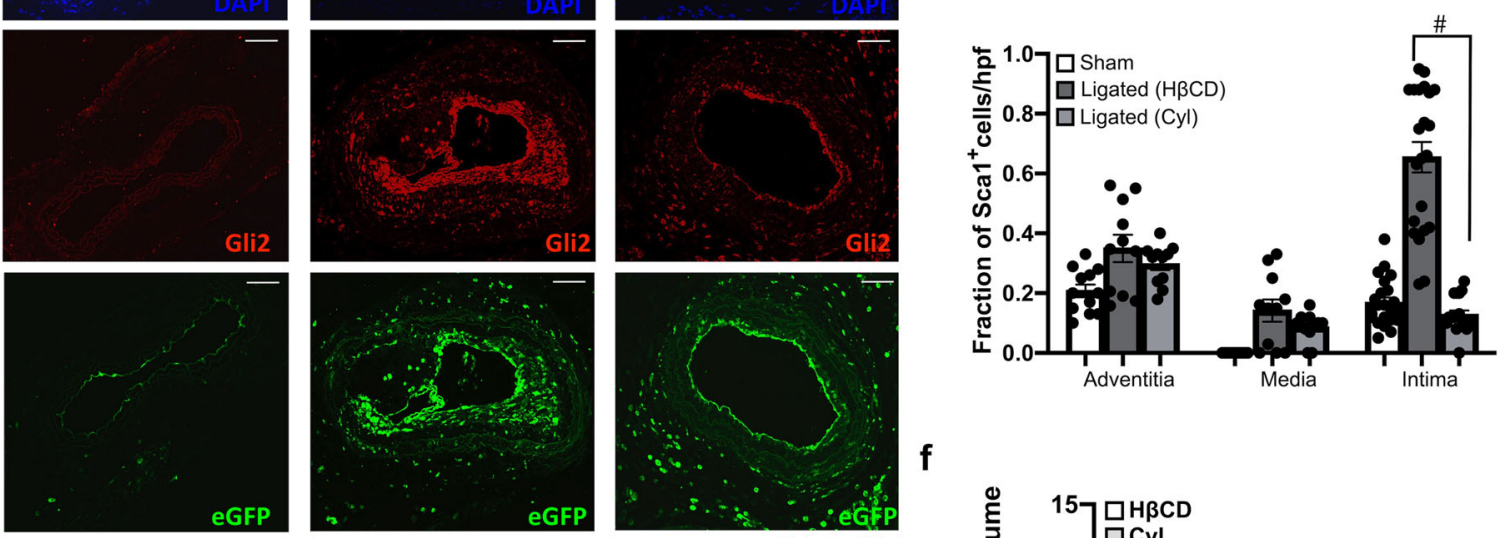

d
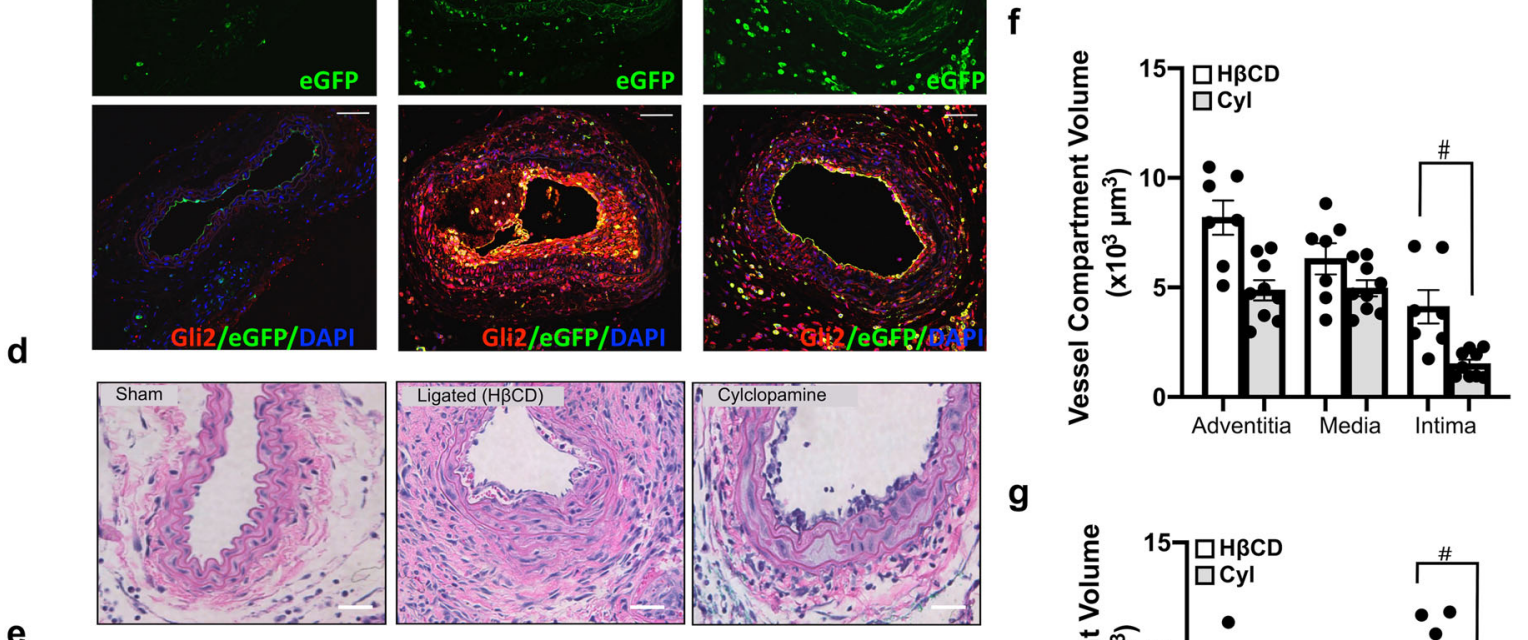

g
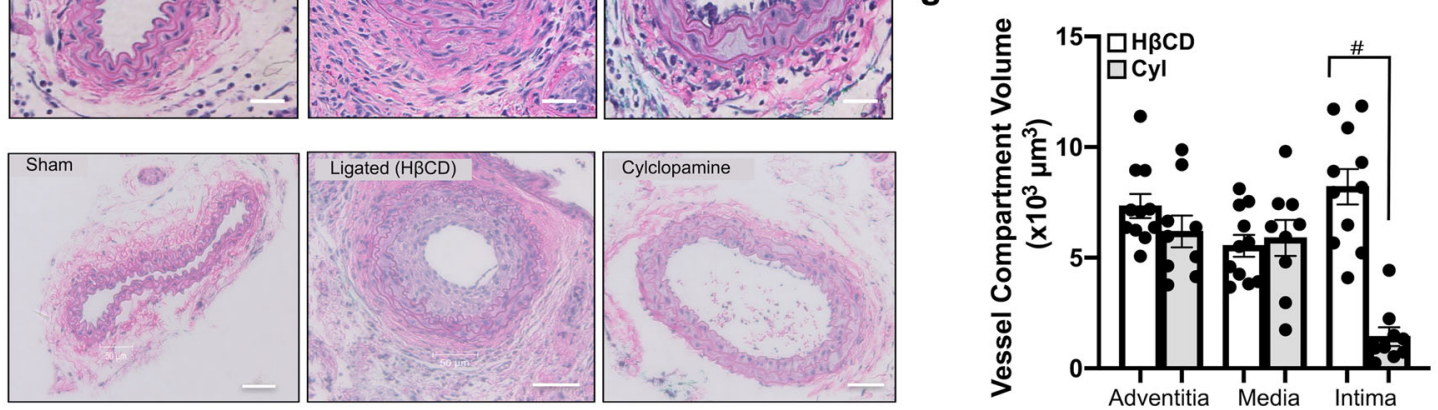

Fig. 4 Inhibition of $\mathrm{Hh}$ signalling attenuates intimal thickening and Sca1 cell expansion following iatrogenic flow restriction. a Representative immunofluorescence images of Hh receptor patched 1 (Ptch1) (red), Hh target gene Gli2 (red), Sca1-eGFP (green) expression and merged (eGFP/Gli2/DAPI) in carotid artery cross sections from sham-operated, ligated vehicle control (H $\beta C D)$, and ligated + cyclopamine $(10 \mathrm{mg} / \mathrm{kg}, \mathrm{IP})$ treated mice after 14 days. Scale bar $=$ top panel: $50 \mu \mathrm{m}$, all other panels: $20 \mu \mathrm{m}$. b, c Cumulative data showing the fraction of Sca1-eGFP cells within the adventitia, media and intima from sham-operated, ligated vehicle control (H $\beta C D)$, and ligated + cyclopamine $(C y l)$ Sca1-eGFP mice after $\mathbf{b} 7$ days and $\mathbf{c} 14$ days. Data are the mean \pm SEM of five sections per experimental group, \# $p \leq 0.05$ vs ligated $\mathrm{H} \beta C D$ controls and are representative of a minimum of four animals per experimental group. $\mathbf{d}$, e Representative H\&E staining of carotid artery cross sections from sham, ligated control (H $\beta C D$ vehicle), and ligated + cyclopamine (Cyl) treated animals after $7 \mathrm{~d}(\mathbf{d})$ and e $14 \mathrm{~d}$. Scale bar $=50 \mu \mathrm{m}$. f, g Morphometric analysis of vessel compartment volumes for ligated control (H $\beta C D)$, and ligated + cyclopamine (Cyl) treated animals after f $7 \mathrm{~d}$ and $\mathbf{g} 14 \mathrm{~d}$. Data mean \pm SEM of $7-12$ sections analysed from four animals, ${ }^{\#} p \leq 0.05$ vs ligated control (H $\left.\beta C D\right)$. 

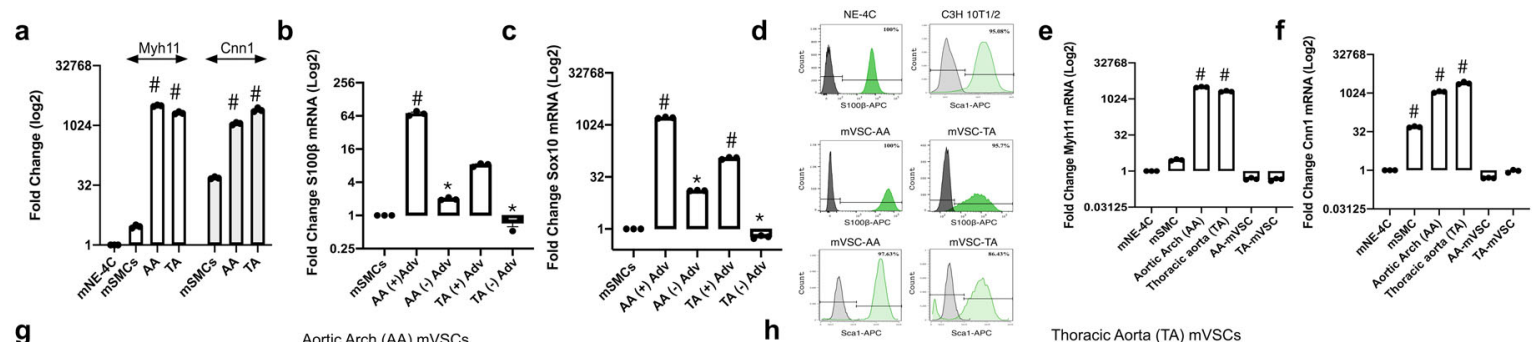

g
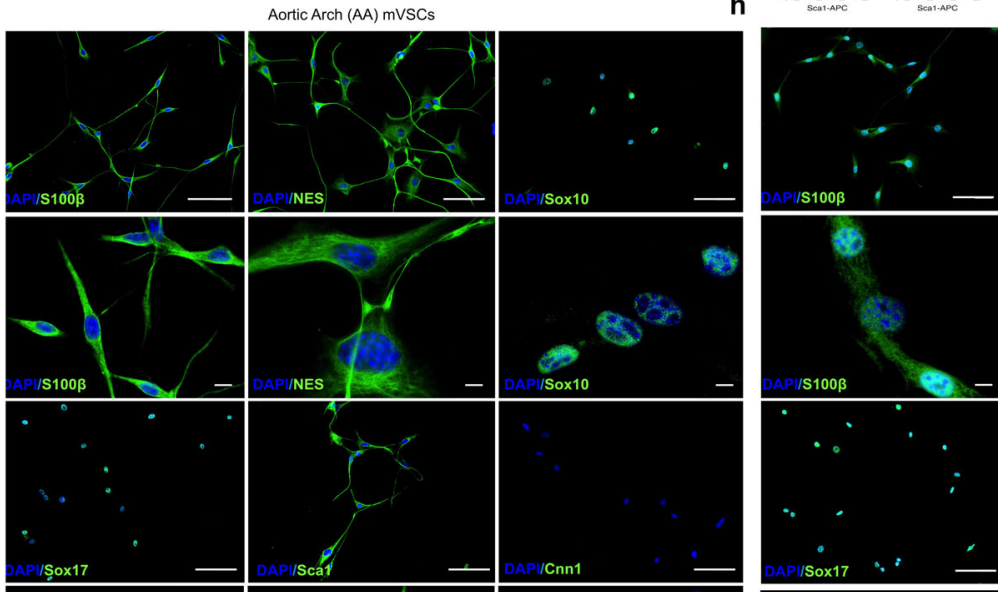

Thoracic Aorta (TA) mVSCs
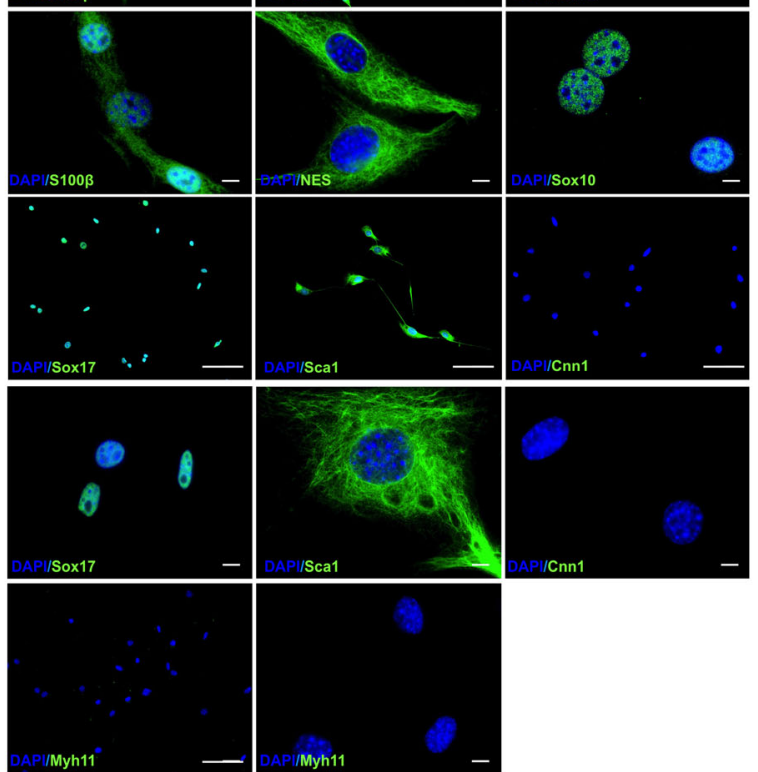

I $\mathbf{m}$
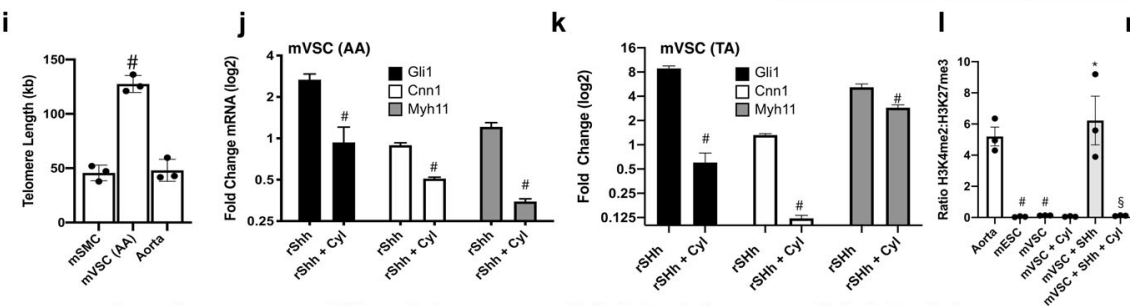

j

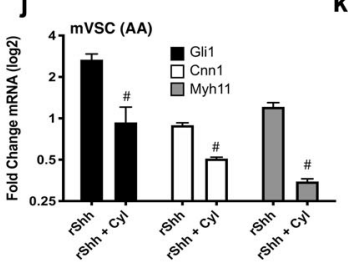

SHh $(0.5 \mu \mathrm{g} / \mathrm{ml})$

Differentiation
Media (DM)

O Control
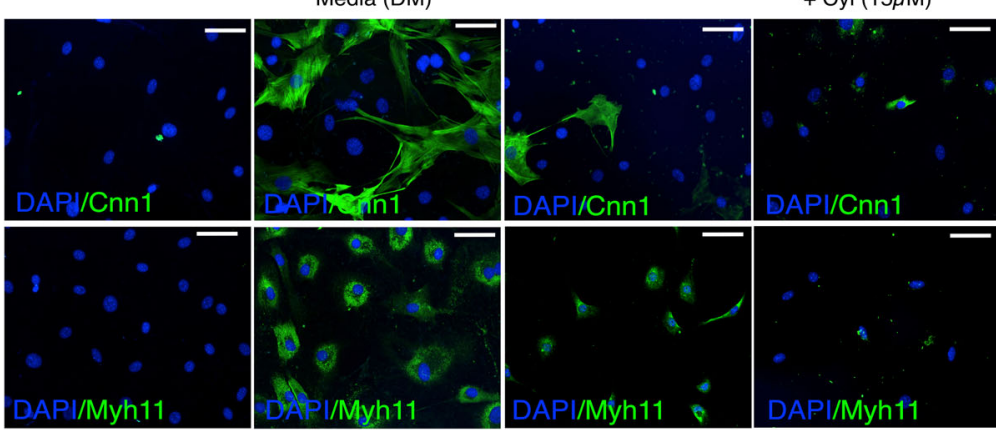

DAPI/Mýh11

Hedgehog signalling promotes myogenic differentiation of resident murine $S 100 \beta / S c a 1$ vascular stem cells in vitro

The functional effect of sonic hedgehog (SHh) signalling on the growth and myogenic differentiation of vSCs was evaluated in vitro using multipotent $\mathrm{Sca} 1 / \mathrm{S} 100 \beta$ cells isolated from mouse aorta. Whole-vessel Gli1 mRNA levels increased over time following ligation [Supplementary Fig. $6 c$ ]. Treatment of vSCs isolated from AA and TA with recombinant SHh $(\mathrm{rSHh}, 0.5 \mu \mathrm{g} / \mathrm{ml}$, $24 \mathrm{~h}$ ) promoted $\mathrm{Hh}$ signalling by increasing Hh target gene mRNA levels (Gli1, Gli2 and Ptch1), an effect blocked following pre- 
Fig. 5 Resident $S 100 \beta / S c a 1$ vascular stem cells from atheroprone and atheroresistant regions of the mouse aorta in vitro. a Relative levels of Myh11 and Cnn1 in AA and TA regions of the mouse aorta. Data are expressed as the Log2 fold change in mRNA levels relative to neural stem cells (NE-4C) in culture and are the mean \pm SEM of three aortic specimens, ${ }^{\#} p \leq 0.05$ vs NE-4C cells. The housekeeping gene Gapdh was used as a control. b, c The level of enrichment of mRNA for neuroectodermal markers b S100 $\beta$ and $\mathbf{c}$ Sox 10 within atheroprone AA (aortic arch) and atheroresistant TA (thoracic/descending aorta) regions of the mouse aorta in the absence or presence of the adventitial (Adv) layer. The housekeeping gene hypoxanthine guanine phosphoribosyltransferase (hprt) was used as a control. Data are expressed as the Log2 fold change in mRNA levels relative to murine aortic SMCs in culture and are the mean \pm SEM of three aortic specimens, " $p \leq 0.05$ vs MOVAS SMCs. d Flow cytometry analysis of vSCs isolated from AA and TA regions of the mouse aorta and grown in maintenance media for S100 $\beta$ (dark green) and Sca1 (light green) expression. NE-4C and CH3-10T1/2 cells were used as positive controls, respectively. Filled dark and light green curves represent negative control samples. e, $\mathbf{f}$ Relative levels of e Myh11 and $\mathbf{f}$ Cnn 1 in vSCs isolated from AA and TA regions of the mouse aorta, compared to AA and TA aortic tissue. Data are expressed as the Log2 fold change in mRNA levels relative to neural stem cells (NE-4C) in culture and are the mean \pm SEM of three independent cultures and three aortic specimens, $\# \leq 0.05$ vs NE- $4 \mathrm{C}$ cells. g, h Representative immunocytochemical analysis of stem cell markers S100 $\beta$, Nestin, Sox10, Sox17, Sca1, and SMC differentiation markers, Cnn1 and Myh11 in vSCs isolated and grown in maintenance media (MM) from $\mathbf{g ~ A A}$ and $\mathbf{h}$ TA regions of the mouse aorta. Scale bar $20 \mu \mathrm{m}$. i Absolute telomere length of vSCs compared to cultured MOVAS SMC and fresh aortic tissue. The telomere primer set recognizes and amplifies telomere sequences. The single copy reference (SCR) primer set recognizes and amplifies a $100 \mathrm{bp}$-long region on mouse chromosome 10, and serves as reference for data normalization. Data are expressed as absolute telomere length $(\mathrm{kb})$ and are the mean \pm SEM of three plates and aortic specimens, $" p \leq 0.05$ vs MOVAs SMCs. j, k Relative levels of Gli1, Myh11 and Cnn1 within vSCs isolated from $\mathbf{j}$ AA and $\mathbf{k}$ TA regions of the mouse aorta in the absence or presence of $\mathrm{rSHh}(0.5 \mu \mathrm{g} / \mathrm{ml})$ with or without the smoothened inhibitor, cyclopamine $(10 \mu \mathrm{M})$. Data are expressed as the Log2 fold change in mRNA levels relative to vSCs alone (control) and are the mean \pm SEM of three representative wells from two independent experiments, ${ }^{*} p \leq 0.05$ versus rSHh alone. I-m ChiP analysis of the ratio of I H3K4me2:H3K27me3 and m H3K27me3:H3K4me2 enrichment at the Myh11 locus in AA vSCs in the absence or presence of $\mathrm{rSHh}(0.5 \mu \mathrm{g} / \mathrm{ml})$ with or without cyclopamine $(10 \mu \mathrm{M})$ for $7 \mathrm{~d}$. $\mathbf{n}$ Fold enrichment of H3K4me2 at the Myh11 locus in AA vSCs in the absence or presence of $\mathrm{rSHh}(0.5 \mu \mathrm{g} / \mathrm{ml})$ with or without cyclopamine (15 $\mu \mathrm{M})$ for $7 \mathrm{~d}$. Fresh aortic tissue and mouse ECSs was used as positive and negative controls, respectively Data are the mean \pm SEM, $n=3, \# p \leq 0.05$ versus aorta/ESC, ${ }^{*} p \leq 0.05$ vs vSC, ${ }^{\S} p \leq 0.05$ vs $\mathrm{rSHh}$. o Representative immunocytofluorescence staining and $\mathbf{p}$ the fraction of Cnn 1 and Myh11 positive cells in the absence or presence of $\mathrm{rSHh}(0.5 \mu \mathrm{g} / \mathrm{ml})$ with or without cyclopamine $(15 \mu \mathrm{M})$ for 7 days. Representative images shown, Scale bar $=50 \mu \mathrm{m}$. Cumulative data is the mean $\pm \mathrm{SEM}, n=3,{ }^{*} p \leq 0.05$ versus control, ${ }^{*} p \leq 0.05$ vs rSHh.

treatment with cyclopamine [Fig. 5j, k and Supplementary Fig. 6a, b]. Concurrent treatment of cells with rSHh for 7 days resulted in a significant increase in SMC differentiation marker gene expression (Cnn1 and Myh11), an effect attenuated by pre-treatment of cells with cyclopamine [Fig. 5j, k]. SHh treatment also significantly increased the growth of AA vSCs after 7 days, an effect attenuated by pre-treatment with cyclopamine [Supplementary Fig. $6 \mathrm{~d}$ ].

The level of enrichment of the SMC specific epigenetic histone mark, di-methylation of lysine 4 on histone $\mathrm{H} 3$ (H3K4me2) and the repressor mark, tri-methylation of lysine 27 on histone $\mathrm{H} 3$ (H3K27me3) at the Myh11 locus was assessed using chromatin immunoprecipitation (ChIP) assays before and after rSHh treatment. Freshly isolated differentiated aortic medial SMC and murine embryonic stem cells (mESCs) were used as positive/ negative controls for each mark and were markedly enriched for H3K4me2 and H3K27me3 at the Myh11 promoter, respectively [Fig. 5I-n]. In contrast to medial SMCs, AA vSCs were enriched for H3K27me3 but lacked enrichment of H3K4me2 at this locus. Treatment with recombinant $\mathrm{SHh}(\mathrm{rSHh}, 0.5 \mu \mathrm{g} / \mathrm{ml}, 7 \mathrm{~d})$ increased the enrichment levels of the $\mathrm{H} 3 \mathrm{~K} 4 \mathrm{me} 2$ mark while concurrently decreasing the enrichment of the H3K27me3 mark, an effect attenuated by cyclopamine [Fig. 5I-n]. Finally, SHh treatment significantly increased the fraction of $\mathrm{Cnn}^{+}$and Myh $11^{+}$cells in AA vSCs, an effect attenuated by pre-treatment with cyclopamine [Fig. 5o, p].

\section{S100 $\beta$ cells populate human arteriosclerotic lesions}

The expression of SMA, S100 $\beta$, and the hedgehog receptor, PTCH1, was determined by immunofluorescence staining in normal and arteriosclerotic human aortic sections. The expression of SMA was abundant in the medial layers of both normal and arteriosclerotic vessels while reduced within the neointima [Fig. $6 a]$. The expression of $S 100 \beta$ was confined to the adventitial layer of normal vessels but was significantly enhanced within the adventitial and intimal layers of arteriosclerotic sections [Fig. 6a]. The expression of PTCH1 was confined to the adventitial layers of normal vessels but was also sparsely expressed in the medial and intimal layers of arteriosclerotic sections [Fig. 6a].

\section{Hedgehog signalling promotes myogenic differentiation of human induced pluripotent stem cell (HiPSC)-derived neuroectodermal $S 100 \beta$ vascular stem cells in vitro}

To address whether human neuroectoderm $\mathrm{S} 100 \beta$ progenitors (NEPs) respond to hSHh by undergoing myogenic differentiation, $S 100 \beta$ NEPs were isolated from human induced pluripotent stem cells (HiPSC) using a chemically defined protocol ${ }^{43}$. Human iPSCs enriched for Oct4 were treated with neuroectodermal inductive stimuli (FGF2 and the selective inhibitor of ALK5, SB413642) for 7 days [Fig. $6 \mathrm{~b}$ ] and expressed $\mathrm{S} 100 \beta$ but not SMC differentiation markers CNN1 and MYH11 [Fig. 6b]. Treatment of NEPs with myogenic media supplemented with TGF- $\beta 1 / P D G F-B B$ significantly increased the fraction of CNN1 and MYH11 cells after a further $12 \mathrm{~d}$ in culture, while concomitantly decreasing the number of $S 100 \beta$ cells [Fig. $6 c$ ]. The levels of $S 100 \beta, C N N 1$, and $\mathrm{MYH} 11$ transcripts mirrored the changes in protein, where NEPderived SMCs became significantly enriched for CNN1 and MYH11 following TGF- $\beta 1 /$ PDGF-BB treatment as they diminish their expression of $\mathrm{S} 100 \beta$ [Fig. 6d]. Treatment of NEPs with $\mathrm{rSHh}$ $(1.0 \mu \mathrm{g} / \mathrm{ml})$ for $12 \mathrm{~d}$ resulted in a significant increase in CNN1 and MYH11 mRNA levels, an effect attenuated by cyclopamine [Fig. 6e, $\mathrm{f}]$, concomitant with an increase in the number of CNN1-positive cells [Fig. 6g]. SHh treatment also significantly increased the growth of $\mathrm{S} 100 \beta$ NEPs after 12 days in culture, an effect attenuated by pre-treatment with cyclopamine [Supplementary Fig. 6e].

\section{DISCUSSION}

In this study, we used transgenic mice and genetic lineage tracing approaches to delineate the involvement of Hh-responsive S100 $\beta$ resident vSCs in progressing neointima formation following flow restriction. Our findings indicate that intimal thickening involves significant accumulation of $\mathrm{Hh}$-responsive $\mathrm{S} 100 \beta$-derived progeny. Elucidation of this new cellular $S 100 \beta$ source for generating SMClike cells following injury expands our current understanding of vascular pathology and may represent an important therapeutic strategy for combating subclinical atherosclerosis.

SMC-like cells within neointimal lesions reportedly are monoclonal/oligoclonal in origin arising from the expansion of a pre- 
a
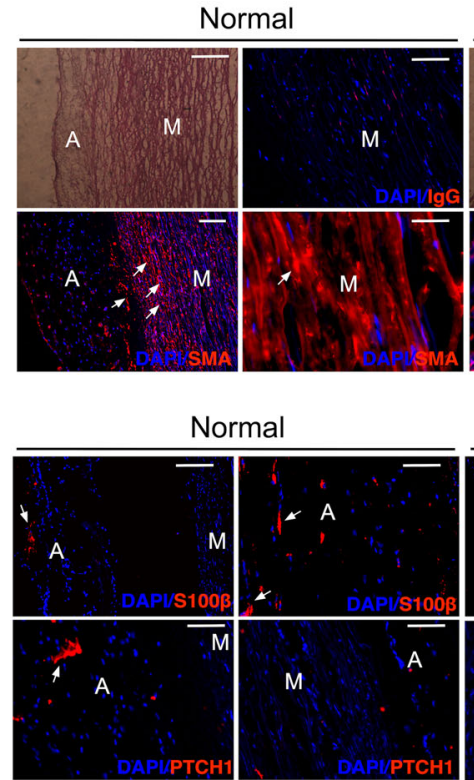

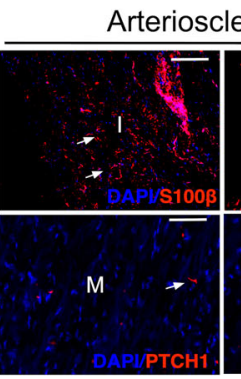

Arteriosclerotic

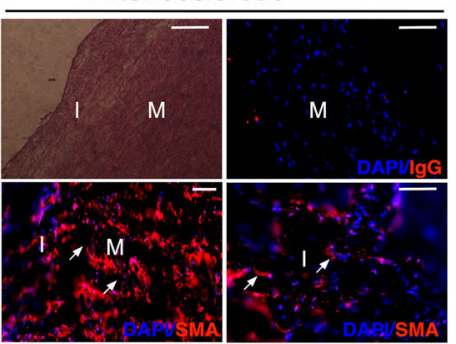

lerotic

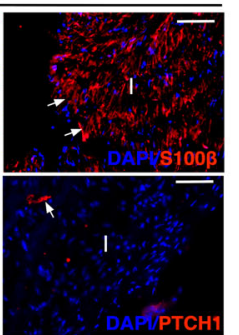

c
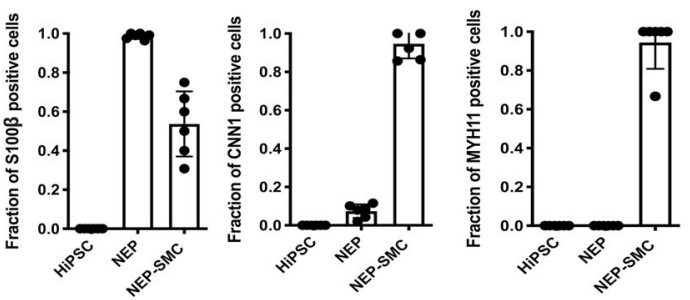

d

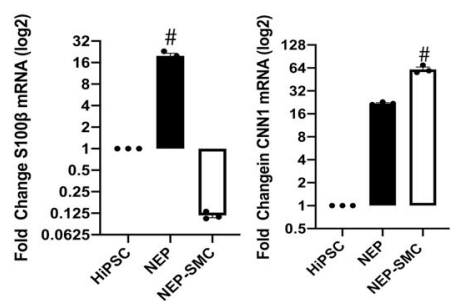

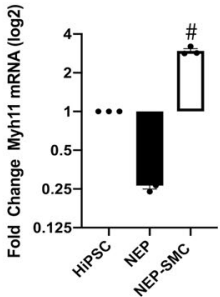

b

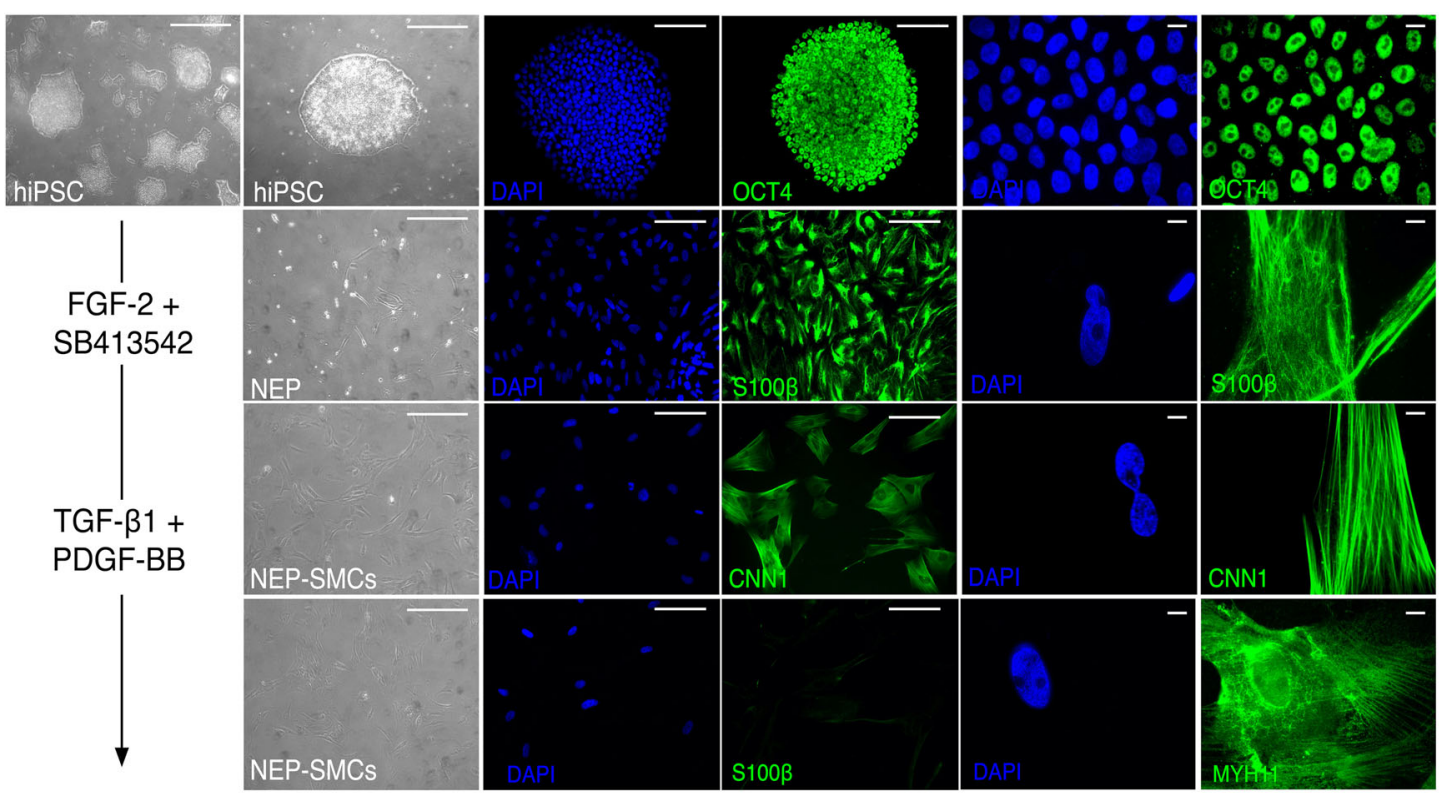

e
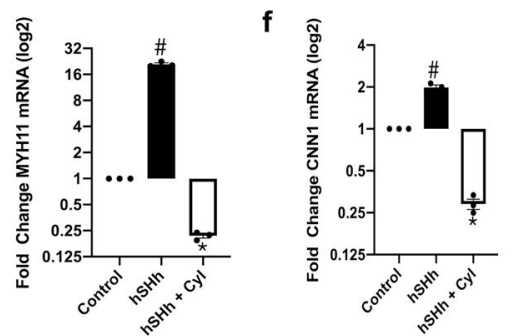

g
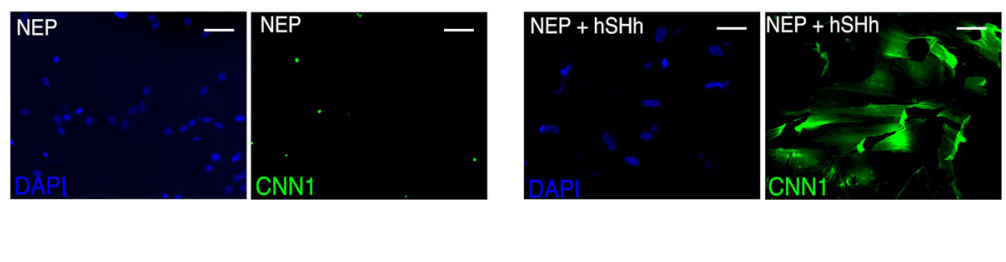

existing resident clonal population of cells ${ }^{44-47}$. Despite extensive research, their origin has remained controversial ${ }^{48}$. As a general rule, SMCs are purported to switch from a contractile phenotype to a less mature synthetic phenotype following vascular injury resulting in the loss of expression of SMC differentiation contractile markers, increased proliferation, and the synthesis and release of pro-inflammatory cytokines, chemotaxis-associated molecules, and growth factors ${ }^{49}$. However, whether these clonal progeny within lesions are derived primarily from differentiated medial SMCs ${ }^{8,9,49}$, endothelial cells ${ }^{10,50}$, and/or resident vSCs ${ }^{10-13}$ remains unclear. Over the last decade, vascular SMC lineage tracing studies have provided compelling evidence of a significant role for pre-existing medial SMCs in vascular pathologies ${ }^{8,51-53}$. Single-cell RNA sequence analysis (scRNA-seq) further identified a rare $\mathrm{Sca}^{+}$Myh11-Cre marked SMC subpopulation that gives rise to functionally distinct neointimal cells during lesion progression 
Fig. 6 Human neuroectoderm progenitors express $S 100 \beta$ cells and are present in vascular lesions. a Representative phase contrast photomicrographs, hematoxylin and eosin (H\&E) staining, DAPI staining (blue) and immunocytochemical staining for smooth muscle cell $\alpha$ actin (SMA) (red), S100 $\beta$ (red) and PTCH1 as indicated in the adventitial (A), medial (M), and intimal (I) sections of normal human and arteriosclerotic arteries. Scale bar $=50 \mu \mathrm{m}$. Data are representative of three sections from three patient cohorts. b Phase contrast images, fluorescence staining of DAPI nuclei (blue) and immunoctytochemical analysis of the expression of OCT4 (green), S100 $\beta$ (green) in NEPs and CNN1 (green) and MYH11 (green) in NEP-derived SMCs. All cells were generated as described previously ${ }^{43}$. Scale bar = Right panels: $50 \mu \mathrm{m}$, Left panels: $20 \mu \mathrm{m}$. Data are representative of five images per experimental group from three independent cultures. c The fraction of S100 $\beta$,

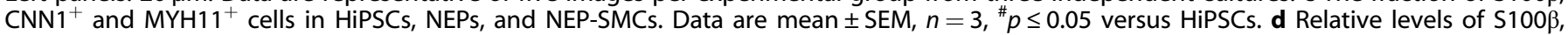
CNN1 and MYH11 in HiPSCs, NEPs and NEP-SMCs, respectively. Data are expressed as the Log2 fold change in mRNA levels relative to HiPSCs in culture and are the mean \pm SEM $, n=3,{ }^{\#} p \leq 0.05$ versus HiPSCs. e, $\mathbf{f}$ Relative levels of e CNN1 and $\mathbf{f}$ MYH11 in NEPs in the absence or presence of hSHh $(0.5 \mu \mathrm{g} / \mathrm{ml})$ with or without cyclopamine $(10 \mu \mathrm{M})$ for $12 \mathrm{~d}$. Data are representative of three independent cultures, ${ }^{\#} p \leq 0.05$ versus NEPs, ${ }^{*} p \leq 0.05$ vs hSHh. g Immunoctytochemical analysis of CNN1 (green) expression and DAPI (blue) staining in NEPs in the absence or presence of recombinant hSHh $(0.5 \mu \mathrm{g} / \mathrm{ml})$ for $12 \mathrm{~d}$. Data are representative of five images per experimental group from two independent cultures.

in mice ${ }^{8,9}$. However, although the Myh11-CreERT2 transgene was originally deemed specific for vascular $\mathrm{SMC}^{54}$, data indicating expression of this and other 'SMC differentiation genes' in nonSMC populations has recently emerged ${ }^{55-57}$ raising the possibility that other resident cells may be inadvertently marked and contribute to lesion formation. Myh11-CreER SMC lineage tracing studies have also demonstrated that the fraction of SMCs originating from pre-existing SMCs may be considerably smaller after severe transmural injury in mice further suggesting a contributory role for cells other than medial $\mathrm{SMCs}^{58}$.

In this study, we provide genetic evidence that vascular lesions contain an abundance of dual labelled Hh-responsive S100 $/$ Sca 1 vSCs derived from a non-SMC perivascular and/or medial S100 $\beta$ parent population. Notwithstanding the fact that Sca1 cells are present in bone marrow ${ }^{59}$, bone marrow cells were not marked using the $\mathrm{S} 100 \beta$-CreERT2-tdT. It is therefore most likely that Sca1/ S100 $\beta$ cells marked with S100 $\beta$-CreERT2-tdT within lesions were locally derived ${ }^{47}$. S100 $\beta$ cells predominate within the adventitial layer of healthy vessels (murine and human) since removal of the adventitia dramatically reduced aortic $S 100 \beta$ transcript levels in mice. Nevertheless, a small fraction of $\mathrm{S} 100 \beta / \mathrm{Sca} 1^{+}$cells are present in medial layer of normal vessels and could be successfully isolated from aortic medial segments following enzymatic digestion and sequential plating in vitro. Indeed, previous studies using in situ immunohistochemical analysis and scRNA-seq also confirms the presence of a sparse Sox $10 / S 100 \beta$ cell population within the media, in addition to the adventitia ${ }^{9,60,61}$. However, these enrichment levels were minimal compared to an $\mathrm{S} 100 \beta^{+}$ neuronal cell and vascular adventitial 'fibroblast-like cell' phenotypes within the vessel wall, supporting the contention that a perivascular adventitial $S 100 \beta$ cell may be the predominant source of $S 100 \beta$ within the vessel wall. Similarly, a discrete population of immature medial cells expressing CD146 that emerge during embryonic development may also contribute to neointimal formation in response to injury ${ }^{58}$. Whether these CD146 immature SMC progenitors are also $\mathrm{S} 100 \beta / \mathrm{Sca} 1$ remains unclear although $S 100 \beta$ vSCs derived from rat and murine aortic explants express $C D 146^{12,42}$. In the future, it will be important to determine whether medial S100 $\beta$ vSCs differ from CD146 ${ }^{58}$ and Myh11-CreER Sca1 cells ${ }^{9}$ in their propensity to modulate different phenotypes and undergo clonal expansion using various injury models.

Adventitial progenitors have long been implicated in the progression of neointimal lesions following various vascular injuries $^{62,63}$. However, in the absence of Sca1 or c-kit ${ }^{+}$stem cell lineage tracing experiments in vivo, the hypothesis of a regenerative function for adventitial Sca1 or c-kit ${ }^{+}$vSCs in lesion formation has been difficult to prove until recently. While perivascular cells must first traverse the internal and external elastic lamina to accumulate within the intima following injury ${ }^{64}$, disruption of the elastic lamellae and flow-dependent enlargement of fenestrae can facilitate such migration ${ }^{65,66,67}$. Cell fate mapping studies have now clarified the role for adventitial
PDGFRa Sca1 progenitors in lesion progression following severe injury ${ }^{11}$ while c-kit progenitors minimally facilitate endothelial regeneration or SMC differentiation to $S M C s^{68}$. As Sca1-CreER marked cells were not present in vascular lesions following wireinduced injury ${ }^{11}$, the origin of Sca1 neointimal cells following flow restriction in the current study is unlikely to be from a Sca1 parent population despite expressing Sca1 (albeit using a different injury model). Indeed, the percentage of perivascular S100 $\beta$-CreER marked cells that were $\mathrm{Sca}^{+}$in normal vessels was $<50 \%$ indicating that a Sca $1^{\text {neg }} / \mathrm{S} 100 \beta^{\text {pos }}$ population might be responsible. In this context, $S 100 \beta$ has been shown to maintain an intermediate state of Sca1 progenitor cells following lesion formation ${ }^{20}$. Alternatively, this residual $S c a 1^{\text {pos }} / S 100 \beta^{\text {neg }}$ population of adventitial Sca1 cells might represent Sca1 cells derived from differentiated Myh11-CreER SMCs that expand in number following injury and contribute to adventitial remodelling ${ }^{14}$ and lesion formation ${ }^{9}$. While Sca1/S100 $\beta$ cells were predominantly found in the developing intimal layer after 7, 14, and 21 days, these cells were also present in the medial layer following injury. In agreement with previous studies demonstrating significant apoptosis of medial SMCs following flow restriction ${ }^{69}$, the appearance of medial Sca1/S100 $\beta$ cells marked with S100 $\beta$-CreERT2-tdT following early expression of the proapoptotic protein Bax within the media is consistent with regeneration of medial SMCs following injury.

Previous lineage tracing studies have also implicated endothelial cells (EC) that undergo endothelial-to-mesenchymal transition (EndMT) to SMC-like cells as contributing to neointimal formation during vein graft remodelling ${ }^{10,50}$. EndMT promotes neointimal hyperplasia and induces atherogenic differentiation of $\mathrm{EC}^{70}$. In the current study, EC were not marked with $\mathrm{S} 100 \beta$-CreERT2-tdT prior to flow restriction suggesting that the $S 100 \beta$-CreERT2-tdT marked cells within lesions do not originate from an EC parent population. These apparent differences may result from the use of different models of vascular injury where vein graft and atherosclerosis models involve different biomechanical stimuli and/or a different inflammatory environments compared to the flow restriction model $^{50,71}$. While EC did not contribute to neointimal formation in developed lesions, a potential role for adluminal Sca1 cells in regenerating the endothelium following ligation was observed. As flow restriction leading to alterations in shear stress and cyclic strain is strongly associated with increased endothelial cell apoptosis in vitro ${ }^{72}$ and in vivo ${ }^{73}$, significant Bax expression at the adluminal surface was observed after flow restriction. Bcl-2/ Bax ratios are an important rheostat to determine the incidence of apoptosis in general and increases in Bax expression have been widely reported and validated using the TUNNEL assay to indicate apoptosis of endothelial cells in vitro in response to low shear stress (LSS) ${ }^{74}$ and in vivo following carotid artery ligation in wildtype mice ${ }^{75}$ or following flow perturbation in ApoE deficient mice $^{76}$. The increase in Bax expression concomitant with an increase in the number of Sca1 cells that maintain a CD31 and 
eNOS endothelial cell phenotype at the adluminal surface is consistent with the appearance of new endothelial cells in the injured vessel that are derived from the proliferation of adluminal Sca1 cells. Indeed, recent cell fate mapping studies using Sca1CreERT2-tdT mice have since confirmed this observation by demonstrating expansion of Sca1-CreERT marked cells in the intimal layer that maintain $\mathrm{CDH} 5$ endothelial cell fate following injury ${ }^{11}$.

There are many potential reasons for the different outcomes of lineage tracing studies that addressed the origin of lesional SMClike cells including technical considerations around fixation, tissue preservation, brightness and contrast settings for image acquisition, autofluorescent material such as lipofuscin and single-cell resolution ${ }^{77}$. Another important reason may involve the use of tamoxifen (Tm) in time-sensitive Tm-dependent Cre-LoxP models. Tm-induced Cre recombinase has been shown to indelibly mark cells up to 4 weeks after the last Tm injection thereby potentially confounding the interpretation of many previous studies ${ }^{41}$. In this context, we found that the fraction of S100ß-CreER (tdT) marked cells within the various compartments of ligated vessels four weeks after the last Tm injection was similar to that observed after one week, indicating that, at least in our hands, medial SMC that might undergo de-differentiation and acquire the $S 100 \beta$ promoter are not being inadvertently marked after injury.

Several GWAS studies have implicated HHIPL1 at the chromosome $14 q 32$ CAD locus ${ }^{27,78}$ and identified it as a secreted $\mathrm{Hh}$ signaling protein that modulates atherosclerosis-relevant vascular phenotypes ${ }^{28}$. Here we provide direct evidence that inhibition of Hh signalling controls neointimal thickening following flow restriction by attenuating the volume and accumulation of Sca1 cells within lesions. Furthermore, Hh signalling directly promotes Sca1/S100 $\beta$ cells from mouse and S100 $\beta$ neuroectoderm stem cells derived from human induced pluripotent stem cells (HiPSC) to undergo growth and myogenic differentiation to SMC-like cells in vitro. The possibility that Hh-responsive S100ß/ Sca1 cells isolated from murine AA and TA regions are derived from a Myh11 medial SMC population was ruled out by examining the H3K4me2 epigenetic profile of $\mathrm{S} 100 \beta / \mathrm{Sca} 1$ cells at the Myh11 locus, which is preserved during SMC phenotypic modulation in vitro and in vivo following injury ${ }^{79}$. Hh-responsive $S 100 \beta / S c a 1$ vSCs do not have this SMC histone mark and only become enriched following myogenic differentiation to SMC-like cells, an effect attenuated by the $\mathrm{Hh}$ inhibitor, cylopamine. The accumulation of Sca1/S100 $\beta$ cells within lesions may be due, in part, to asymmetric division of Sca1/S100 $\beta$ progenitor stem cells (from the adventitia and/or media) and subsequent proliferation and migration of their partially differentiated myogenic progeny to the intima. Indeed, SHh promotes $\mathrm{Sca} 1 / \mathrm{S} 100 \beta$ growth and the proliferation of modulated, partially differentiated SMCs in vitro ${ }^{32}$. Moreover, previous studies have shown that it is a Sca1 adventitial population in particular that proliferate following injury using immunohistochemical co-staining with $\mathrm{Ki}-67^{31}$. This is not surprising as adventitial Sca1 stem cells co-localise with $\mathrm{SHh}$ and its receptor, Ptch $1^{35}$ while several previous studies support a role for

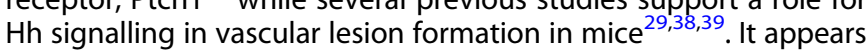
the Yes-Associated Protein (YAP) is required for incorporation and expansion of Sca1-derived neointimal cells following injury ${ }^{11}$ where the Hh pathway reportedly acts upstream of the Hippo pathway to regulate stem cell maintenance ${ }^{80}$. Indeed, our data suggest $S 100 \beta$ cells are also present in normal vessels within the adventitial layer where $\mathrm{Hh}$ components reside ${ }^{81,82}$. In arteriosclerotic lesions, $\mathrm{S} 100 \beta$ expression is exaggerated within the neointimal layer with sparse staining for PTCH1 present. As S100 $\beta$ NEP-derived SMCs are considered crucial to the generation of SMC in vascular lesions within atheroprone regions of the murine vasculature $^{4,43}$, it is tempting to speculate that Hh-responsive S100ß-derived SMC-like progeny may also play a pivotal role in lesion formation in humans. Future studies that address the interactions between Hippo pathway and Hh may yield therapeutic strategies aimed at interfering with YAP activity through inhibition of hedgehog pathway, which downregulates the YAP protein $^{83,84}$.

In conclusion, the identification of a population of $\mathrm{S} 100 \beta /$ Sca1 cells within vascular lesions that are derived from a non-SMC $S 100 \beta$ parent population is novel and highlights these cells as an important source for intimal thickening following injury. Furthermore, the role of $\mathrm{SHh}$ in mediating this process expands our understanding of the mechanisms contributing to the progression of vascular lesions. While $\mathrm{Hh}$ signalling has previously been implicated in the development of vascular lesions through modulation of a differentiated SMC phenotype ${ }^{28}$, our study further suggests that $\mathrm{Hh}$ signalling may also control myogenic differentiation and accumulation S100ß/Sca1-derived SMC-like progeny within vascular lesions. While hedgehog signalling appears critical, further research is required to investigate the precise mechanisms driving migration, proliferation and differentiation of stem cells during lesion formation. It is possible that SMC-like cells within lesions comprise several different stages of the myogenic, osteogenic, and myeloid differentiation of various stem cells and hence display a variety of divergent phenotypes. The elucidation of these mechanisms will provide vital information needed for the development of more efficient and novel therapies for vascular proliferative diseases. Our results provide a potential strategy for the treatment of atherosclerosis by combating cell migration and asymmetric differentiation of a perivascular-derived $S 100 \beta$ parent vSC population.

\section{METHODS}

\section{Mice breeding and genotyping}

All procedures were approved by the University of Rochester Animal Care Committee in accordance with the guidelines of the National Institutes of Health for the Care and Use of Laboratory Animals. Sca1-eGFP transgenic mice were obtained from JAX labs; Stock \#012643, strain name B6.Cg.Tg (Ly6a-EGFP)G5Dzk/j. These transgenic mice have an enhanced green fluorescent protein (eGFP) under the control of murine lymphocyte antigen 6 complex, locus A (Ly6a) promoter. Hemizygous Ly6a-GFP mice are viable, fertile, normal in size and do not display any gross physical or behavioural abnormalities ${ }^{85}$.

S100ß-EGFP/Cre/ERT2 transgenic mice (JAX Labs, stock \#014160, strain name B6;DBA-Tg(S100ß-EGFP/cre/ERT2)22Amc/j) express the eGFPCreER ${ }^{\mathrm{T} 2}$ (Enhanced Green Fluorescent Protein and tamoxifen inducible cre recombinase/ESR1) fusion gene under the direction of the mouse S100 $\beta$ promoter. Ai9 mice (Jax Labs, stock \#007909, strain name B6.Cg-Gt(ROSA) 26 Sor $^{\text {tm9(CAG-tdTomato)Hze } / J) ~ e x p r e s s ~ r o b u s t ~ t d T o m a t o ~ f l u o r e s c e n c e ~ f o l l o w-~}$ ing Cre-mediated LoxP recombination. For lineage tracing experiments S100ß-eGFP/Cre/ERT2-dTomato double transgenic mice of both genders were generated by crossing $\mathrm{S} 100 \beta-\mathrm{eGFP} / \mathrm{CreERT} 2$ mice with Ai9 reporter mice. The tdTomato transgene expression pattern corresponds to genomic marked $\mathrm{S} 100 \beta$ cells, and the eGFP transgene expression pattern corresponds to constitutive expression of $S 100 \beta$. Mice were genotyped using genomic DNA prepared from tail samples. All male and female mice were included in the study and were 8-10 weeks old.

\section{Tamoxifen-induced genetic lineage tracing}

S100ß-CreER-Rosa26tdT mice (average weight $20 \mathrm{~g}, 6-8$ weeks old) were injected IP with tamoxifen (Tm) dissolved in corn oil at $75 \mathrm{mg} / \mathrm{kg}$ for 5 consecutive days. Carotid artery ligation (partial, or complete), or sham operation, was performed at 1 week or 4 weeks after the last injection of $\mathrm{Tm}$. At the indicated time post-ligation or sham operation, anesthetized mice were perfusion fixed and carotid arteries harvested for analysis.

\section{Carotid artery ligation}

Ligation of the left common carotid artery was performed essentially as described previously ${ }^{38} 1$ and 4 weeks after Tm-induced cre-recombination, in male and female S100 $\beta$-eGFP/Cre/ERT2-dTomato double transgenic mice. Prior to surgery mice received a single dose of Buprenorphine SR (sustained release) analgesia (0.5-1.0 mg/kg SQ) (and every $72 \mathrm{~h}$ thereafter 
as needed). The animal was clipped and the surgical site prepped using betadine solution and alcohol. A midline cervical incision was made. For partial carotid artery ligation, with the aid of a dissecting microscope, the left external and internal carotid arterial branches were isolated and ligated with 6-0 silk suture reducing left carotid blood flow to flow via the patent occipital artery. The neck incision (2 layers, muscle and skin) was sutured closed. Partial ligation of the left carotid artery in this manner resulted in a decrease ( $80 \%)$ in blood flow, leaving an intact endothelial monolayer. Buprenorphine was administered at least once post-op 6-12 h. For complete ligation, the left common carotid was isolated and ligated just beneath the bifurcation with 6-0 silk suture. In sham surgery group, carotid was similarly manipulated but not ligated. The neck incision (2 layers, muscle and skin) was sutured closed and the animal allowed recover under observation. After 3, 7, 14, and 21 days, mice were perfused with $4 \%$ PFA via the left ventricle for $10 \mathrm{~min}$, and carotid arteries were harvested for analysis.

\section{Cyclopamine treatment}

Sca1-eGFP mice were treated with the smoothened inhibitor, cyclopamine, or the vehicle 2-hydropropyl- $\beta$-cyclodextrin $(\mathrm{H} \beta C D$ ) (Sigma-Aldrich) alone as a control, (essentially as described previously) ${ }^{86}$. Mice were injected ( $250 \mu$ l max volume) intraperitoneally (IP) 1 day before ligation, then every other day after ligation at a dose of $10 \mathrm{mg} / \mathrm{kg}$ Cyclopamine (Sigma-Aldrich) dissolved in a solution of $45 \%(w / v) H \beta C D$. There was no effect of cyclopamine treatment on mouse body weight, compared to the $\mathrm{H} \beta C D$ vehicle control group (data not shown).

\section{Histomorphometry}

At specified times post-ligation, mice were anesthetized (ketamine/ xylazine) and perfusion fixed with $4 \%$ paraformaldehyde in sodium phosphate buffer ( $\mathrm{pH}$ 7.0). Fixed carotids were embedded in paraffin for sectioning. Starting at the carotid bifurcation landmark (single lumen) a series of cross sections $(10 \times 5 \mu \mathrm{m})$ were made, every $200 \mu \mathrm{m}$ through $2 \mathrm{~mm}$ length of carotid artery. Cross sections were de-paraffinized, rehydrated in graded alcohols and stained with Verhoeff-Van Gieson stain for elastic laminae and imaged using a Nikon TE300 microscope equipped with a Spot RT digital camera (Diagnostic Instruments). Digitized images were analyzed using SPOT Advanced imaging software. Assuming a circular structure in vivo, the circumference of the lumen was used to calculate the lumen area, the intimal area was defined by the luminal surface and internal elastic lamina (IEL), the medial area was defined by the IEL and external elastic lamina (EEL), and the adventitial area was the area between the EEL and the outer edge, essentially as described by us previously ${ }^{38}$.

\section{Haematoxylin and eosin staining of tissue}

For human artery cross sections, slides were placed in ice-cold acetone and held at $-20^{\circ} \mathrm{C}$ for $15 \mathrm{~min}$. They were then washed $3 \times$ PBS before immersion in Harris Haematoxylin at room temperature for $8 \mathrm{~min}$. Slides were processed by sequential washing-in distilled water for $5 \mathrm{~min}$; $1 \%$ acid alcohol for $30 \mathrm{sec}$; $0.1 \%$ sodium bicarbonate for $1 \mathrm{~min}$, and $95 \%$ ethanol. Slides were then washed in Eosin for $1 \mathrm{~min}, 75 \%$ ethanol for $3 \mathrm{~min}$, $95 \%$ ethanol for $3 \mathrm{~min}$, and $100 \%$ ethanol for $3 \mathrm{~min}$ before being submerged in Histoclear for 3 min and permanently mounted using three drops of DPX, cleaned with $100 \%$ ethanol before analysis.

For murine vessels, paraffin rehydration was conducted at room temperature by immersing the slides in xylene for $20 \mathrm{~min}$. The slides were then immersed in the following gradients of ethanol for $5 \mathrm{~min}$, respectively: $100,90,70$, and $50 \%$ ethanol. The slides were then rinsed in distilled $\mathrm{H}_{2} \mathrm{O}_{2}$ before washing in $1 x$ PBS for $10 \mathrm{~min}$. The slides were stored in $1 \times$ PBS until ready when they were immersed in Harris Haematoxylin at room temperature for $8 \mathrm{~min}$.

S100 $\beta^{+}$cells were identified as either "genomically marked" red tdTomato expressing cells or "currently expressing" green fluorescent protein (eGFP) cells visualized in de-paraffinized S100ß-eGFP/Cre/ ERT2-dTomato mouse carotid cross sections mounted with Sigma Fluoroshield with DAPI, using an FV1000 Olympus or a Nikon A1R HD laser scanning confocal microscope. Numbers of red or green fluorescent cells and DAPI nuclei (blue) in carotid cross section images from different experimental groups were either analyzed by Fiji ImageJ software in each vessel compartment using a grid system.

\section{Whole mount histochemical analysis}

Briefly, arteries harvested from transgenic mice were washed in PBS before transfer to $10 \%$ neutral buffered formalin and incubated at $4{ }^{\circ} \mathrm{C}$ overnight. The tissue was rinsed $3 \times$ PBS before mounting on histology slides using DAPI containing fluoroshield (Sigma). The whole tissue was then imaged using a Nikon Eclipse TS100 inverted microscope, EXFO X-Cite ${ }^{\mathrm{TM}} 120$ fluorescence system and assessed using SPOT imaging software.

\section{Immunofluorescent staining of tissues}

Immunostaining essentially as previously described ${ }^{38}$. Carotid artery cryosections were air-dried for $1 \mathrm{~h}$ at room temperature, followed by incubation with blocking buffer (5\% donkey serum, $0.1 \%$ Triton X-100 in PBS) for $30 \mathrm{~min}$ at room temperature and then incubated with primary antibody overnight at $4{ }^{\circ} \mathrm{C}$ in antibody solution (2.5\% BSA, $0.3 \mathrm{M}$ Glycine and $1 \%$ Tween in DPBS). Murine and human arterial sections were stained with primary antibodies [Supplementary Table I]. Isotype IgG control and secondary antibody only controls were performed. For antigen retrieval, slides were brought to a boil in $10 \mathrm{mM}$ sodium citrate $(\mathrm{pH}$ 6.0) then maintained at a sub-boiling temperature for $10 \mathrm{~min}$. Slides were cooled on the bench-top for $30 \mathrm{~min}$ then washed in deionized water $(3 \times 5 \mathrm{~min})$ each before being washed in PBS $(3 \times 5 \mathrm{~min})$. The antigen retrieval protocol diminishes endogenous eGFP and tdT tomato transgene signals. Therefore, those sections were co-stained with anti-eGFP antibody and anti-Td tomato antibody [Supplementary Table I].

For immunofluorescence staining, 5 consecutive images were obtained and processed using ImageJ software ${ }^{\mathrm{TM}}$ to analyze the collected images. Mouse carotid artery cross sections were mounted with fluoroshield with DAPI and analyzed using an FV1000 Olympus or a Nikon A1R HD laser scanning confocal microscope. Images were merged using the Image color-merge channels function. Merged signals and split channels were used to delineate the signals at single-cell resolution. Settings were fixed at the beginning of both acquisition and analysis steps and were unchanged. Brightness and contrast were lightly adjusted after merging.

\section{Immunocytofluorescent staining of cells}

Cells seeded onto UV sterilized coverslips were fixed with $3.7 \%$ formaldehyde, $(15 \mathrm{~min}, \mathrm{RT})$. If cells required permeabilization for the detection of intracellular antigens, cells were incubated in $0.025 \%$ Triton X100 PBS (room temp, $15 \mathrm{~min}$ ). All coverslips were blocked (1 h, RT) using $5 \%$ BSA, $0.3 \mathrm{M}$ Glycine, 1\% Tween PBS solution (1 h, RT). Cells were incubated with primary antibodies overnight at $4{ }^{\circ} \mathrm{C}$ [Supplemental Table II], then washed twice with PBS to remove any unbound primary antibody before being incubated $(1 \mathrm{~h}, \mathrm{RT})$ with the recommended concentration of fluorochrome-conjugated secondary antibodies diluted in blocking buffer [Supplementary Table II]. Following $2 \times$ wash in PBS, cell nuclei were stained using DAPI: PBS (dilution 1:1000) (15 min, RT). For each primary and secondary antibody used, a secondary control and an $\lg G$ isotype control was performed to assess nonspecific binding. An Olympus CK30 microscope and FCell ${ }^{T M}$ software was used to capture images. Images were analyzed using ImageJ software as described above. Settings were fixed at the beginning of both acquisition and analysis steps and were unchanged. Brightness and contrast were lightly adjusted after merging.

\section{Cell culture and harvesting}

Murine aortic SMCs (MOVAS (ATCC ${ }^{\circledR}$ CRL-2797 $7^{\text {TM}}$ ) were cultured in Dulbeco's Modified Eagles growth medium (DMEM) supplemented with Fetal Bovine Serum (10\%), L-glutamine (2 mM, Gibco), and PenicillinStreptomycin ( $100 \mathrm{U} / \mathrm{mL}$, Gibco). Murine neuroectodermal stem cells (mNE4Cs, ATCC ${ }^{\circledR}$ CRL-2925 ${ }^{T M}$ ) were grown in Eagle's Minimum Essential Medium supplemented with Fetal Bovine Serum (10\%), L-glutamine (2 mM, Gibco) and Penicillin-Streptomycin $(100 \mathrm{U} / \mathrm{mL}$, Gibco) using Poly-L-Lysine coated plates $(15 \mu \mathrm{g} / \mathrm{ml})$. Murine embryonic C3H $10 \mathrm{~T} 1 / 2$ cells (ATCC ${ }^{\circledR}$ CRL-226 ${ }^{\text {TM }}$ ) were grown in Eagle's Basal medium supplemented with heat-inactivated fetal bovine serum to a final concentration of $10 \%$ and $2 \mathrm{mM} \mathrm{L-glutamine.}$ Murine embryonic stem cells (mESCs) ES-D3 [D3] (ATCC ${ }^{\circledR}$ CRL-1934 ${ }^{\mathrm{TM}}$ ) were maintained in culture in Mouse ES-Cell Basal Medium (ATCC SCRR-2011) supplemented with 2-mercaptoethanol $(0.1 \mathrm{mM})$ and $15 \%$ ES-Cell Qualified FBS (ATCC SCRR-30-2020) and a $1000 \mathrm{U} / \mathrm{mL}$ mouse leukemia inhibitory factor (LIF) (Millipore Cat. No. ESG1107). Murine vSCs from AA and TA regions of the mouse aorta were grown in DMEM media supplemented with chick embryo extract (2\%), FBS embryonic qualified (1\%, ATCC), B-27 Supplement, serum free, N-2 Supplement (Cell Therapy Systems), 
recombinant mouse bFGF $(0.02 \mu \mathrm{g} / \mathrm{mL})$, 2-Mercaptoethanol $(50 \mathrm{nM})$, retinoic acid $(100 \mathrm{nM})$, Penicillin-Streptomycin $(100 \mathrm{U} / \mathrm{mL}$, Gibco) (MM).

\section{Isolation of S100ß/Sca1 murine resident vascular stem cells (vSCs)}

Using an optimised enzymatic cell dissociation technique protocol vSCs from both atheroprone (aortic arch-neuroectodermal, NE), and atheroresistant (thoracic aorta-paraxial mesodermal, PM) regions of the murine aorta were isolated using sequential seeding on adherent and non-adherent plates. Mouse thoracic aortas ( 4 at a time) were harvested and placed in cold Hank's solution for adipose tissue removal and lumen rinsing. The adventitia was enzymatically removed by incubation of the vessels in collagenase solution of MEM-a containing GlutaMAX ${ }^{\mathrm{TM}}(2 \mathrm{ml})$, collagenase type $1 \mathrm{~A}(0.7 \mathrm{mg} / \mathrm{ml})$, soybean trypsin inhibitor $(50 \mathrm{mg} / \mathrm{ml})$, and bovine serum albumin $(1 \mathrm{mg} / \mathrm{ml})$ for approx. $10-20 \mathrm{~min}$ at $37^{\circ} \mathrm{C}$. Once the adventitia became lose, it was carefully removed as an intact layer using forceps under a dissecting microscope. The aorta was divided into aortic arch (AA) and thoracic aorta (TA) regions and cut into $1 \mathrm{~mm}$ pieces and digested with Elastase type III (Sigma) at $37^{\circ} \mathrm{C}$. Dispersed cells were centrifuged and washed twice in warm maintenance medium (MM) (DMEM supplemented with $2 \%$ chick embryo extract, $1 \% \mathrm{FBS}, 0.02 \mu \mathrm{g} / \mathrm{ml}$, bFGF basic protein, B-27 Supplement, N-2 supplement, $1 \%$ PenicillinStreptomycin, $50 \mathrm{nM}$ 2-Mercaptoethanol, and $100 \mathrm{nM}$ retinoic acid) before seeding (1st seeding) on a 6-well non-adherent plate in MM. At $48 \mathrm{~h}$, suspension cells were transferred (2nd seeding) to a fresh well of a CELLstart $^{\mathrm{TM}}$ pre-coated 6-well plate in MM. Additional MM was added to the remaining cells in the non-adherent surface. Cells were incubated at $37^{\circ} \mathrm{C}$, $5 \% \mathrm{CO}_{2}$ for 1 week with minimal disturbance. The stem cells exhibited a uniform neural-like morphology in low density culture adopting a dendritic-like tree shape and retaining their morphological characteristics at low density throughout repeated passage. Cells were fed with MM every 2-3 days and passaged every 3-4 days or when $~ 70 \%$ confluent.

\section{Generation of human neuroectoderm progenitors (NEPs) from induced pluripotent stem cells (HiPSCs)}

Human induced pluripotent stem cells (HiPSC) were obtained from HipSci (Cambridge, UK) and cultured per the manufacturer's instructions. Briefly, human dermal fibroblasts were isolated from excess skin of patients undergoing plastic surgery and reprogrammed as previously described ${ }^{87}$. HiPSCs were grown on plates pre-coated using Vitronectin $(10 \mu \mathrm{g} / \mathrm{ml})$ and maintained in complete Essential eight medium replacing $95 \%$ of the medium daily. A ROCK inhibitor $(10 \mu \mathrm{M})$ was added to optimize colony growth. Cells were subcultured with $0.05 \mathrm{mM}$ EDTA for 4-8 min at room temperature until colonies displayed bright halos around the edges and small holes appeared throughout. Cells were frozen down in knockout serum solution with $10 \%$ DMSO. HiPSCs at $70 \%$ confluency were routinely split at a ratio of 1:10 and cells were used between passage $20-40$.

For neuroectoderm differentiation, cells were grown in chemically defined neuroectodermal medium (CDM) containing IMDM, F12 nutrient mix, chemically defined lipid concentrate, transferrin, monothioglycerol, penicillin-streptomycin and $0.5 \mathrm{~g}$ of PVA, and insulin, as previously described ${ }^{43}$. Neuroectoderm differentiation was initiated in CDM supplemented with FGF2 $(12 \mathrm{ng} / \mathrm{ml})$ and SB431542 $(10 \mu \mathrm{M})$ for 7 days. Neuroectodermal (NE) progenitors were maintained in CDM-PVA supplemented with FGF2 $(12 \mathrm{ng} / \mathrm{ml})$ and SB431542 $(10 \mu \mathrm{M})$ and subcultured using TrypLE ${ }^{\mathrm{TM}}$ for $3-5 \mathrm{~min}$ at $37^{\circ} \mathrm{C}$ in a $5 \% \mathrm{CO}_{2}$ incubator. NE progenitors were used between passage 1-10.

To initiate myogenic SMC differentiation, NEPs were subcultured in SMC differentiation medium containing $\mathrm{CDM}^{+}$PDGF-BB $(10 \mathrm{ng} / \mathrm{ml}$, PeproTech) + TGF- $\beta 1$ ( $2 \mathrm{ng} / \mathrm{ml}$, PeproTech) for 12 days. Neuroectoderm-derived SMCs (NESMC) were maintained in CDM supplemented with $10 \%$ FBS. After $12 \mathrm{~d}$ of PDGF-BB and TGF- $\beta 1$ treatment, all cells appeared spindle-shaped. Complete medium was then changed to DMEM 10\% FBS, $1 \%$ Penicillin-streptomycin for expansion. Cells were frozen down using $90 \%$ (volume/volume) $\mathrm{FBS}^{+} 10 \%$ (vol/vol) DMSO. The NE-SMC were used between passage 3-20.

\section{Human tissue}

Tissue sections from paired normal human aorta and arteriosclerotic vessels were obtained from Amsbio LLC, tissue repository.

\section{Chromatin immunoprecipitation}

ChIP was performed on cultured cells as previously described with slight modifications ${ }^{79}$. Cells at $70 \%$ confluency were fixed with $1 \%$ paraformaldehyde (10 min, RT). Cross-link was stopped by addition of $125 \mathrm{mM}$ glycine for $10 \mathrm{~min}$. The cross-linked chromatin was sonicated to shear chromatin into fragments of 200-600 base pairs. The sheared chromatin was immunoprecipitated with $2 \mu \mathrm{g}$ tri-methyl-histone $\mathrm{H} 3$ (Lys27) and dimethyl-histone $\mathrm{H} 3$ (Lys4), while negative control was incubated with mouse IgG and input DNA without antibody using the CHIP-IT Express HT Kit from Active Motif (Cat no: 53018) according to the manufacturer's instructions [Supplementary Table III]. A chromatin IP DNA Purification Kit (Cat No 58002 Active Motif) was used to purify the samples after CHIP before PCR was performed using Myh11 primers [5' -CCC TCC CTT TGC TAA ACA CA-3' and 5' - CCA GAT CCT GGG TCC TTA CA-3]. Sensimix SYBR ${ }^{\circledR}$ noROX Bioline Kit (QT650) was used to perform Real Time One-Step PCR according to the manufacturers' instructions. The antibodies used for ChIP are outlined in Supplemental Table I.

\section{Quantitative PCR}

Total RNA was prepared from cultured cells using the ReliaPrep ${ }^{\text {TM }}$ RNA Cell Miniprep System kit from Promega according to the manufacturer's protocol. Two micrograms of RNA was used for reverse transcription with Rotor-Gene SYBR Green RT-PCR (QIAGEN) or The SensiMix ${ }^{\text {TM }}$ SYBR $^{\circledR}$ No-ROX (BioLine) protocols for Real TimeOne-Step RT-PCR using the Real Time Rotor-GeneRG-3000 ${ }^{\text {TM }}$ light cycler from Corbett Research using primers listed in Supplementary Table IV.

\section{Flow cytometry and analysis}

Isolated cells were incubated with I fluorochrome-conjugated antibodies and control lgG's or (ii) the appropriate primary antibody with fluorochrome-conjugated secondary antibodies at a final concentration of $1 \mu \mathrm{g}$ per sample and incubated at $4^{\circ} \mathrm{C}$ for $30 \mathrm{~min}$ [Supplementary Table V]. Fluorescence labeled cells analysis was performed using a BD FACS Aria flow cytometry system (BD Biosciences), and data were analyzed with FlowJo $^{\text {TM }}$ software (Tree Star, Ashland, Ore) and De Novo software FCS Express 4 Flow Cytometry (Pasadena, CA).

\section{Telomere assay}

The average telomere length in cells was measured using a ScienCell's Absolute Mouse Telomere Length Quantification qPCR Assay Kit (AMTLQ) according to the manufacturer's instructions. The telomere primer set recognizes and amplifies telomere sequences and is designed to directly measure the average telomere length of murine cells.

\section{Quantification and statistical analysis}

All data were determined from multiple individual biological samples and presented as mean values \pm standard error of the mean (SEM). All in vitro experiments were performed in triplicate and repeated three times unless otherwise stated. All mice were randomly assigned to groups (including both male and female), and the analysis was performed blind by two groups. For statistical comparisons, all data were checked for normal gaussian distribution before parametric and non-parametric tests were performed. An unpaired two-sided Student's $t$-test was performed using GraphPad Prism software $v 9^{\mathrm{TM}}$ for comparing differences between two groups, and ANOVA test for over multiple comparisons. Significance was accepted when $p \leq 0.05$.

\section{Reporting summary}

Further information on research design is available in the Nature Research Reporting Summary linked to this article.

\section{DATA AVAILABILITY}

The data, materials, and reagents generated or analysed that support the findings of this study are available from the corresponding authors (P.A.C. and E.M.R.) on reasonable request.

Received: 5 August 2020; Accepted: 14 January 2021; Published online: 01 March 2021 


\section{REFERENCES}

1. Bennett, M. R., Sinha, S. \& Owens, G. K. Vascular smooth muscle cells in atherosclerosis. Circ. Res. 118, 692-702 (2016).

2. Sakamoto, A. et al. Pathologic intimal thickening: are we any closer to understand early transitional plaques that lead to symptomatic disease? Atherosclerosis 274, 227-229 (2018).

3. Halcox, J. P. J. et al. Endothelial function predicts progression of carotid intimamedia thickness. Circulation 119, 1005-1012 (2009).

4. Sinha, S., lyer, D. \& Granata, A. Embryonic origins of human vascular smooth muscle cells: implications for in vitro modeling and clinical application. Cell. Mol. Life Sci. 71, 2271-2288 (2014).

5. Korshunov, V. A. \& Berk, B. C. Flow-induced vascular remodeling in the mouse: a model for carotid intima-media thickening. Arterioscler. Thromb. Vasc. Biol. 23, 2185-2191 (2003).

6. Liu, W., Redmond, E. M., Morrow, D. \& Cullen, J. P. Differential effects of dailymoderate versus weekend-binge alcohol consumption on atherosclerotic plaque development in mice. Atherosclerosis 219, 448-454 (2011).

7. Nam, D. et al. Partial carotid ligation is a model of acutely induced disturbed flow, leading to rapid endothelial dysfunction and atherosclerosis. Am. J. Physiol. Heart Circ. Physiol. 297, H1535-H1543 (2009).

8. Chappell, J. et al. Extensive proliferation of a subset of differentiated, yet plastic, medial vascular smooth muscle cells contributes to neointimal formation in mouse injury and atherosclerosis models. Circ. Res. 119, 1313-1323 (2016).

9. Dobnikar, L. et al. Disease-relevant transcriptional signatures identified in individual smooth muscle cells from healthy mouse vessels. Nat. Commun. 9, 4567 (2018).

10. Yuan, F. et al. Contribution of vascular cells to neointimal formation. PLOS ONE 12, e0168914 (2017).

11. Tang, J. et al. Arterial Sca $1^{+}$vascular stem cells generate de novo smooth muscle for artery repair and regeneration. Cell Stem Cell 26, 81-96.e4 (2020).

12. Tang, Z. et al. Differentiation of multipotent vascular stem cells contributes to vascular diseases. Nat. Commun. 3, 875 (2012).

13. Kramann, R. et al. Adventitial MSC-like cells are progenitors of vascular smooth muscle cells and drive vascular calcification in chronic kidney disease. Cell Stem Cell 19, 628-642 (2016).

14. Majesky, M. W. et al. Differentiated smooth muscle cells generate a subpopulation of resident vascular progenitor cells in the adventitia regulated by Klf4. Circ. Res. 120, 296-311 (2017).

15. Wan, $M$. et al. Injury-activated transforming growth factor $\beta$ controls mobilization of mesenchymal stem cells for tissue remodeling. Stem Cells 30, 2498-2511 (2012).

16. Donato, R. et al. S100B's double life: intracellular regulator and extracellular signal. Biochim. Biophys. Acta 1793, 1008-1022 (2009).

17. Cao, T. et al. S100B promotes injury-induced vascular remodeling through modulating smooth muscle phenotype. Biochim. Biophys. Acta Mol. Basis Dis. 1863, 2772-2782 (2017).

18. Sagheddu, R. et al. Targeting RAGE as a potential therapeutic approach to Duchenne muscular dystrophy. Hum. Mol. Genet. 27, 3734-3746 (2018).

19. Zhou, S., Zhu, W., Zhang, Y., Pan, S. \& Bao, J. S100B promotes microglia M1 polarization and migration to aggravate cerebral ischemia. Inflamm. Res. 67, 937-949 (2018).

20. $\mathrm{Wu}, \mathrm{Y}$. et al. S100B is required for maintaining an intermediate state with doublepositive Sca- $1^{+}$progenitor and vascular smooth muscle cells during neointimal formation. Stem Cell Res. Ther. 10, 294 (2019).

21. Petrova, R. \& Joyner, A. L. Roles for Hedgehog signaling in adult organ homeostasis and repair. Development 141, 3445-3457 (2014).

22. Marigo, V., Davey, R. A., Zuo, Y., Cunningham, J. M. \& Tabin, C. J. Biochemical evidence that patched is the hedgehog receptor. Nature 384, 176-179 (1996).

23. Briscoe, J. \& Thérond, P. P. The mechanisms of Hedgehog signalling and its roles in development and disease. Nat. Rev. Mol. Cell Biol. 14, 416-429 (2013).

24. Marigo, V. \& Tabin, C. J. Regulation of patched by Sonic hedgehog in the developing neural tube. Proc. Natl Acad. Sci. USA 93, 9346-9351 (1996).

25. Marigo, V., Johnson, R. L., Vortkamp, A. \& Tabin, C. J. Sonic hedgehog differentially regulates expression of GLI and GLI3 during limb development. Dev. Biol. 180, 273-283 (1996).

26. Chuang, P. T. \& McMahon, A. P. Vertebrate hedgehog signalling modulated by induction of a hedgehog- binding protein. Nature 397, 617-621 (1999).

27. Schunkert, H. et al. Large-scale association analysis identifies 13 new susceptibility loci for coronary artery disease. Nat. Genet. 43, 333-338 (2011).

28. Aravani, D. et al. HHIPL1, a gene at the $14 q 32$ coronary artery disease locus, positively regulates Hedgehog signaling and promotes atherosclerosis. Circulation 140, 500-513 (2019).

29. Beckers, L. et al. Disruption of hedgehog signalling in ApoE - /- mice reduces plasma lipid levels, but increases atherosclerosis due to enhanced lipid uptake by macrophages. J. Pathol. 212, 420-428 (2007).
30. Ali, H. et al. Localization and characterization of a novel secreted protein, SCUBE2, in the development and progression of atherosclerosis. Kobe J. Med. Sci. 59, E122-E131 (2013).

31. Dutzmann, J. et al. Sonic hedgehog-dependent activation of adventitial fibroblasts promotes neointima formation. Cardiovasc. Res. 113, 1653-1663 (2017).

32. Morrow, D. et al. Sonic Hedgehog induces Notch target gene expression in vascular smooth muscle cells via VEGF-A. Arterioscler. Thromb. Vasc. Biol. 29, 1112-1118 (2009).

33. Li, F. et al. Sonic hedgehog signaling induces vascular smooth muscle cell proliferation via induction of the G1 cyclin-retinoblastoma axis. Arterioscler. Thromb. Vasc. Biol. 30, 1787-1794 (2010)

34. Wang, G. et al. Activation of the sonic hedgehog signaling controls human pulmonary arterial smooth muscle cell proliferation in response to hypoxia. Biochim. Biophys. Acta 1803, 1359-1367 (2010).

35. Passman, J. N. et al. A sonic hedgehog signaling domain in the arterial adventitia supports resident $\mathrm{Sca}^{+}$smooth muscle progenitor cells. Proc. Natl Acad. Sci. USA 105, 9349-9354 (2008).

36. Shikatani, E. A. et al. c-Myb regulates proliferation and differentiation of adventitial $\mathrm{Sca}^{+}$vascular smooth Muscle cell progenitors by transactivation of myocardin. Arterioscler. Thromb. Vasc. Biol. 36, 1367-1376 (2016).

37. Tsai, T.-N. et al. Contribution of stem cells to neointimal formation of decellularized vessel grafts in a novel mouse model. Am. J. Pathol. 181, 362-373 (2012).

38. Fitzpatrick, E. et al. Alcohol reduces arterial remodeling by inhibiting sonic Hedgehog-stimulated stem cell antigen-1 positive progenitor stem cell expansion. Alcohol. Clin. Exp. Res. 41, 2051-2065 (2017).

39. Redmond, E. M. et al. Inhibition of patched-1 prevents injury-induced neointimal hyperplasia. Arterioscler. Thromb. Vasc. Biol. 33, 1960-1964 (2013).

40. Fang, Y., Wu, D. \& Birukov, K. G. Mechanosensing and mechanoregulation of endothelial cell functions. Compr. Physiol. 9, 873-904 (2019).

41. Reinert, R. B. et al. Tamoxifen-induced Cre-loxP recombination is prolonged in Pancreatic Islets of adult mice. PLoS ONE 7, e33529 (2012).

42. Kennedy, E. et al. Embryonic rat vascular smooth muscle cells revisited-a model for neonatal, neointimal SMC or differentiated vascular stem cells? Vasc. Cell 6, 6 (2014).

43. Cheung, C., Bernardo, A. S., Trotter, M. W. B., Pedersen, R. A. \& Sinha, S. Generation of human vascular smooth muscle subtypes provides insight into embryological origin-dependent disease susceptibility. Nat. Biotechnol. 30, 165-173 (2012).

44. Murry, C. E., Gipaya, C. T., Bartosek, T., Benditt, E. P. \& Schwartz, S. M. Monoclonality of smooth muscle cells in human atherosclerosis. Am. J. Pathol. 151, 697-705 (1997).

45. Basatemur, G. L., Jørgensen, H. F., Clarke, M. C. H., Bennett, M. R. \& Mallat, Z. Vascular smooth muscle cells in atherosclerosis. Nat. Rev. Cardiol. 16, 727-744 (2019).

46. Gomez, D. \& Owens, G. K. Reconciling smooth muscle cell oligoclonality and proliferative capacity in experimental atherosclerosis. Circ. Res. 119, 1262-1264 (2016).

47. Hoglund, V. J., Dong, X. R. \& Majesky, M. W. Neointima formation: a local affair. Arterioscler. Thromb. Vasc. Biol. 30, 1877-1879 (2010).

48. Nguyen, A. T. et al. Smooth muscle cell plasticity: fact or fiction? Circ. Res. 112, 17-22 (2013)

49. Campbell, J. H. \& Campbell, G. R. Smooth muscle phenotypic modulation-a personal experience. Arterioscler. Thromb. Vasc. Biol. 32, 1784-1789 (2012).

50. Cooley, B. C. et al. TGF- signaling mediates endothelial-to-mesenchymal transition (EndMT) during vein graft remodeling. Sci. Transl. Med. 6, 227ra34-227ra34 (2014).

51. Nemenoff, R. A. et al. SDF-1a induction in mature smooth muscle cells by inactivation of PTEN is a critical mediator of exacerbated injury-induced neointima formation. Arterioscler. Thromb. Vasc. Biol. 31, 1300-1308 (2011).

52. Herring, B., Hoggatt, A. M., Burlak, C. \& Offermanns, S. Previously differentiated medial vascular smooth muscle cells contribute to neointima formation following vascular injury. Vasc. Cell 6, 21 (2014).

53. Shankman, L. S. et al. KLF4-dependent phenotypic modulation of smooth muscle cells has a key role in atherosclerotic plaque pathogenesis. Nat. Med. 21, 628-637 (2015).

54. Owens, G. K. Molecular control of vascular smooth muscle cell differentiation and phenotypic plasticity. Novartis Found. Symp. 283, 174-191 (2007).

55. Chakraborty, R. et al. Promoters to study vascular smooth muscle. Arterioscler. Thromb. Vasc. Biol. 39, 603-612 (2019).

56. Murgai, M. et al. KLF4-dependent perivascular cell plasticity mediates premetastatic niche formation and metastasis. Nat. Med. 23, 1176-1190 (2017).

57. Sheikh, A. Q., Lighthouse, J. K. \& Greif, D. M. Recapitulation of developing artery muscularization in pulmonary hypertension. Cell Rep. 6, 809-817 (2014).

58. Roostalu, U. et al. Distinct cellular mechanisms underlie smooth muscle turnover in vascular development and repair. Circ. Res. 122, 267-281 (2018). 
59. Tobin, S. W. et al. Dual roles for bone marrow-derived Sca-1 cells in cardiac function. FASEB J. 31, 2905-2915 (2017).

60. Tang, Z., Wang, A., Wang, D. \& Li, S. Smooth muscle cells: to be or not to be? Response to Nguyen et Al. Circ. Res. 112, 23-26 (2013).

61. Kalluri, A. S. et al. Single-cell analysis of the normal mouse aorta reveals func tionally distinct endothelial cell populations. Circulation 140, 147-163 (2019).

62. Psaltis, P. J. et al. Characterization of a resident population of adventitial macrophage progenitor cells in postnatal vasculature. Circ. Res. 115, 364-375 (2014).

63. $\mathrm{Hu}, \mathrm{Y}$. et al. Abundant progenitor cells in the adventitia contribute to atherosclerosis of vein grafts in ApoE-deficient mice. J. Clin. Invest. 113, 1258-1265 (2004).

64. Wang, Y.-L., Liu, L.-Z., He, Z.-H., Ding, K.-H. \& Xue, F. Phenotypic transformation and migration of adventitial cells following angioplasty. Exp. Ther. Med. 4, 26-32 (2012).

65. Wong, L. C. Y. \& Langille, B. L. Developmental remodeling of the internal elastic lamina of rabbit arteries. Circ. Res. 78, 799-805 (1996).

66. Guo, Z.-Y., Yan, Z.-Q., Bai, L., Zhang, M.-L. \& Jiang, Z.-L. Flow shear stress affects macromolecular accumulation through modulation of internal elastic lamina fenestrae. Clin. Biomech. 23, S104-S111 (2008).

67. Campbell, G. J. \& Roach, M. R. Fenestrations in the internal elastic lamina at bifurcations of human cerebral arteries. Stroke 12, 489-496 (1981).

68. Chen, Q. et al. Genetic lineage tracing analysis of c-kit+ stem/progenitor cells revealed a contribution to vascular injury-induced neointimal lesions. J. Mol. Cell. Cardiol. 121, 277-286 (2018).

69. Skelly, C. L., Chandiwal, A., Vosicky, J. E., Weichselbaum, R. R. \& Roizman, B. Attenuated herpes simplex virus 1 blocks arterial apoptosis and intimal hyperplasia induced by balloon angioplasty and reduced blood flow. Proc. Natl Acad. Sci. USA 104, 12474-12478 (2007).

70. Moonen, J.-R. A. J. et al. Endothelial-to-mesenchymal transition contributes to fibro-proliferative vascular disease and is modulated by fluid shear stress. Cardiovasc. Res. 108, 377-386 (2015).

71. Souilhol, C., Harmsen, M. C., Evans, P. C. \& Krenning, G. Endothelial-mesenchymal transition in atherosclerosis. Cardiovasc. Res. 114, 565-577 (2018).

72. Pan, L. et al. Shear stress induces human aortic endothelial cell apoptosis via interleukin-1 receptor-associated kinase 2-induced endoplasmic reticulum stress. Mol. Med. Rep. 16, 7205-7212 (2017).

73. Heo, K.-S. et al. PKCZ mediates disturbed flow-induced endothelial apoptosis via p53 SUMOylation. J. Cell Biol. 193, 867-884 (2011).

74. Dong, G. et al. Low shear stress-induced autophagy alleviates cell apoptosis in HUVECs. Mol. Med. Rep. 15, 3076-3082 (2017).

75. Zhang, Y. et al. Protective effect of irisin on atherosclerosis via suppressing oxidized low density lipoprotein induced vascular inflammation and endothelial dysfunction. PLOS ONE 11, e0158038 (2016).

76. Franck, G. et al. Flow perturbation mediates neutrophil recruitment and potentiates endothelial injury via TLR2 in mice: implications for superficial erosion. Circ. Res. 121, 31-42 (2017).

77. Bentzon, J. F. \& Majesky, M. W. Lineage tracking of origin and fate of smooth muscle cells in atherosclerosis. Cardiovasc. Res. 114, 492-500 (2018).

78. van der Harst, P. \& Verweij, N. Identification of 64 novel genetic loci provides an expanded view on the genetic architecture of coronary artery disease. Circ. Res. 122, 433-443 (2018).

79. Gomez, D., Shankman, L. S., Nguyen, A. T. \& Owens, G. K. Detection of histone modifications at specific gene loci in single cells in histological sections. Nat. Methods 10, 171-177 (2013).

80. Fernandez-L, A. et al. YAP1 is amplified and up-regulated in hedgehog-associated medulloblastomas and mediates Sonic hedgehog-driven neural precursor proliferation. Genes Dev. 23, 2729-2741 (2009).

81. Queiroz, K. C. S. et al. Dichotomy in Hedgehog signaling between human healthy vessel and atherosclerotic plaques. Mol. Med. 18, 1122-1127 (2012).

82. Dunaeva, M., van Oosterhoud, C. \& Waltenberger, J. Expression of Hedgehog signaling molecules in human atherosclerotic lesions: an autopsy study. Int. J. Cardiol. 201, 462-464 (2015).

83. Felley-Bosco, E. \& Stahel, R. Hippo/YAP pathway for targeted therapy. Transl. Lung Cancer Res. 3, 75-83 (2014).

84. Cotton, J. L. et al. YAP/TAZ and Hedgehog coordinate growth and patterning in gastrointestinal mesenchyme. Dev. Cell 43, 35-47.e4 (2017).
85. Ma, X., Robin, C., Ottersbach, K. \& Dzierzak, E. The Ly6A Sca1 GFP transgene is expressed in all adult mouse hematopoietic. Stem Cells Stem Cells 20, 514-521 (2002).

86. Van Den Brink, G. R. et al. Sonic hedgehog regulates gastric gland morphogenesis in man and mouse. Gastroenterology 121, 317-328 (2001).

87. Vallier, L. et al. Signaling pathways controlling pluripotency and early cell fate decisions of human induced pluripotent. Stem Cells Stem Cells 27, 2655-2666 (2009).

\section{ACKNOWLEDGEMENTS}

We thank Diana Scott for histological tissue processing, and Drs. Linda Callahan and Paivi Jordan for confocal microscope expertise. This research was funded in part by Science Foundation Ireland grant SFI-11/PI/1128, Health Research Board (HRB) of Ireland grant HRA-POR-2015-1315, the European Union's INTERREG VA Programme, managed by the Special EU Programmes Body (SEUPB) to P.A.C., and NIH R21AA020365 and R21AA023213 to E.M.R., Irish Research Council (IRC) GOIPG/ 2014/43 (M.D.L.) and GOIPG/2016/1529 (E.C.), King Abdulla Scholarship IR15208 ID1066336148 to Y.G.

\section{AUTHOR CONTRIBUTIONS}

E.F., W.L., and J.-C.H., performed the animal experiments and M.D.L. and S.H. performed the blind analysis of the images. E.F. performed the human arteriosclerotic tissue analysis. E.F., M.D.L., R.H., D.B., and E.C., performed the murine and rat cell culture experiments, D.B. performed the HiPSCs cell work. E.C. performed the telomere assay and R.H.J, M.d.L., and Y.G. performed the ChIP analysis. E.M.R., C.L., and P.A.C. drafted the manuscript, revised the manuscript, and discussed the results. E.M. R. and P.A.C. designed and coordinated the experiments, interpreted the data, revised, and confirmed the paper. All authors reviewed and confirmed the manuscript.

\section{COMPETING INTERESTS}

The authors declare no competing interests.

\section{ADDITIONAL INFORMATION}

Supplementary information The online version contains supplementary material available at https://doi.org/10.1038/s41536-021-00120-8.

Correspondence and requests for materials should be addressed to P.A.C.

Reprints and permission information is available at http://www.nature.com/ reprints

Publisher's note Springer Nature remains neutral with regard to jurisdictional claims in published maps and institutional affiliations.

Open Access This article is licensed under a Creative Commons Attribution 4.0 International License, which permits use, sharing, adaptation, distribution and reproduction in any medium or format, as long as you give appropriate credit to the original author(s) and the source, provide a link to the Creative Commons license, and indicate if changes were made. The images or other third party material in this article are included in the article's Creative Commons license, unless indicated otherwise in a credit line to the material. If material is not included in the article's Creative Commons license and your intended use is not permitted by statutory regulation or exceeds the permitted use, you will need to obtain permission directly from the copyright holder. To view a copy of this license, visit http://creativecommons. org/licenses/by/4.0/.

(C) The Author(s) 2021 